\title{
PANDA as midrapidity detector for a future HESR Collider at FAIR
}

\author{
Leonid Frankfurt ${ }^{1}$, Mark Strikman ${ }^{2}$, Alexei Larionov ${ }^{8}$, Andreas Lehrach ${ }^{3,5}$, Rudolf Maier ${ }^{3,5}$, \\ Hendrik van Hees ${ }^{6, a}$, Christian Spieles ${ }^{6}$, Volodymyr Vovchenko ${ }^{4,6}$, Horst Stoecker ${ }^{4,6,7}$ \\ ${ }^{1}$ Sackler School of Exact Sciences, Tel Aviv University, Tel Aviv, Israel \\ 2 Pennsylvania State University, University Park, PA, USA \\ ${ }^{3}$ Institut für Kernphysik, Forschungszentrum Jülich, 52425 Jülich, Germany \\ ${ }^{4}$ Frankfurt Institute for Advanced Studies, Giersch Science Center, 60438 Frankfurt am Main, Germany \\ ${ }^{5}$ JARA-FAME (Forces and Matter Experiments), Forschungszentrum Jülich and RWTH Aachen University, Jülich, Germany \\ ${ }^{6}$ Institut für Theoretische Physik, Goethe Universität Frankfurt, 60438 Frankfurt am Main, Germany \\ ${ }^{7}$ GSI Helmholtzzentrum für Schwerionenforschung GmbH, 64291 Darmstadt, Germany \\ ${ }^{8}$ Institut für Theoretische Physik, Universität Giessen, 35392 Giessen, Germany
}

Received: 28 August 2019 / Accepted: 22 May 2020 / Published online: 23 June 2020

(C) The Author(s) 2020

Communicated by Tamas Biro

\begin{abstract}
Exciting new scientific opportunities are presented for the PANDA detector at the High Energy Storage Ring in the redefined $\bar{p} p(A)$ collider mode, HESR-C, at the Facility for Antiproton and Ion Research (FAIR) in Europe. The high luminosity, $L \sim 10^{31} \mathrm{~cm}^{-2} \mathrm{~s}^{-1}$, and a wide range of intermediate and high energies, $\sqrt{s_{\mathrm{NN}}}$ up to $30 \mathrm{GeV}$ for $\overline{\mathrm{p}} \mathrm{p}(A)$ collisions will allow to explore a wide range of exciting topics in QCD, including the study of the production of excited open charm and bottom states, nuclear bound states containing heavy (anti)quarks, the interplay of hard and soft physics in the dilepton production, probing short-range correlations in nuclei, and the exploration of the early, complete $\overline{\mathrm{p}}$-p- annihilation phase, where an initially pure Yang-Mills gluon plasma is formed.
\end{abstract}

\section{Introduction}

The experimental discovery of charmonium $[2,3]$ and bottomonium [4] in $\mathrm{e}^{+} \mathrm{e}^{-}$and $\mathrm{p} A$ collisions suggests that hadrons containing heavy quarks can be investigated in hadronic processes, where a dense, strongly interacting medium is formed. It can be particularly useful to study the annihilation of antiprotons on free protons and baryons bound in nuclei in $\overline{\mathrm{p}}(A)$ collisions, in both collider and fixed-target experiments at collision energies of $\sqrt{s}=2-200 \mathrm{GeV}$.

The present article is based on the article [1] to be published in the Springer-Nature FIAS Interdisciplinary Science series.

a e-mail: hees@th.physik.uni-frankfurt.de (corresponding author)
A unique opportunity to do this in the near future is provided by the Facility for Antiproton and Ion Research $(\text { FAIR })^{1}$, with the PANDA detector ${ }^{2}$ at the high-energy storage ring (HESR). This concerns both, the presently developed HESR fixed-target mode at $\sqrt{s}<5.5 \mathrm{GeV}$, and a future collider mode at $\sqrt{s}<30 \mathrm{GeV}$, with PANDA as midrapidity detector, see the Appendix for a brief description of the detector.

The collider mode would only need additional proton injection-/beam transfer lines from the SIS18 directly into HESR, as discussed in Ref. [5]. The proton beamline which shall shoot ions and protons clockwise directly into the HESR-Collider shall come down from the transfer beam line connecting the SIS18 with the SIS100. Two switches in both directions for clockwise and counterclockwise (and vice versa) rotating $p$ and $\bar{p}$ beams can be realized in a way similar to the double switch shown as the blue beamline in Fig. 1 , so that both the antiprotons and the protons can be made to circulate the HESR-C counterclockwise and/or clockwise, whereas the PANDA detector can remain nearly unchanged. This allows to eliminate the need for an additional forward arm spectrometer. Fig. 1 also shows the detector areas for

1. atomic and plasma physics, biomedical and material sciences (APPA),

2. the relativistic nucleus-nucleus collision experiments CBM and HADES,

\footnotetext{
${ }_{1}^{1}$ https://fair-center.eu/.

${ }^{2}$ https://panda.gsi.de/oldwww/., https://panda.gsi.de/frontpage
} 


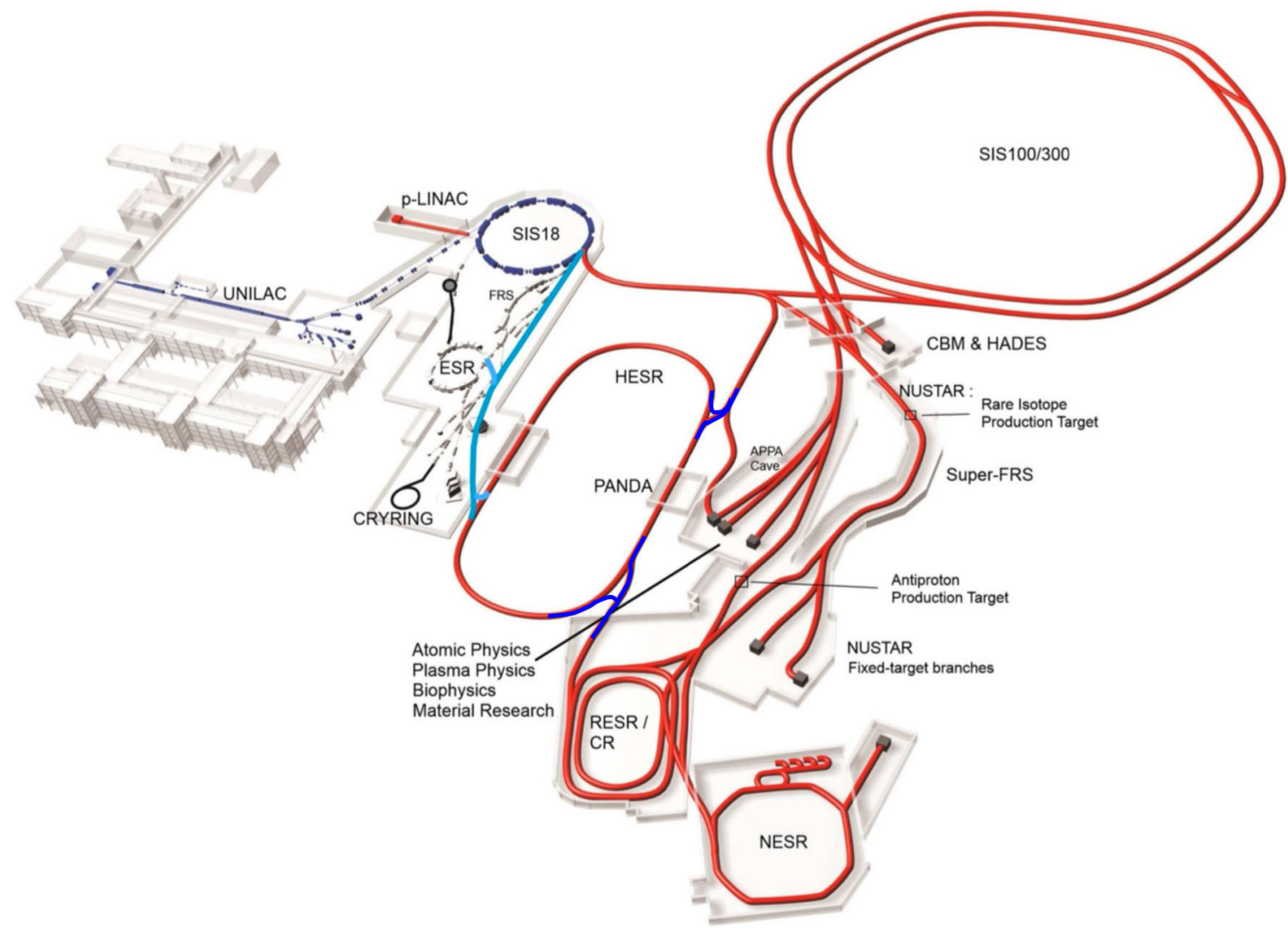

Fig. 1 The existing GSI facility is shown on the left, in dark-blue the existing GSI accelerator facilities and ion sources. On the right the new FAIR complex is displayed in red with its 100 and 300 Tm super-conducting double-ring synchrotrons SIS100 and SIS300 (Fig. adapted from [5])

3. the radioactive beam facility (NUSTAR),

4. the hadron physics detector system PANDA as well as the rare- isotope and antiproton production targets with storage rings.

Proposed beamlines for transport of protons and ions from SIS18 directly to the High Energy Storage Ring HESR [5] and of antiprotons and exotic ions from HESR to the ESR/CRYRING are shown in addition (light blue). To control the systematics and to cover the full range of kinematic acceptance with the PANDA setup it is important that for every injection point of protons and antiprotons into the HESR-C, switches with two injection directions will be installed, such as shown in the dark-blue beamline, so that both the antiprotons and the protons can be made to circulate the HESR-C counterclockwise and/or clockwise.

After the start phase the facility will be completed by the experimental storage rings NESR (New Experimental Storage Ring) and RESR (Recuperated Experimental Storage Ring) enhancing the capabilities of secondary beams and by the superconducting synchrotron SIS300 providing true parallel operation of experimental programs as well as particle energies twentyfold higher compared to those achieved so far at GSI. Additionally, asymmetric HESR collider schemes with somewhat lower center-of-mass energies have been discussed in detail [6-8]. It may also be feasible to study $\overline{\mathrm{p}} A$ collisions for $\sqrt{s_{N N}}$ of up to $19 \mathrm{GeV}$ with interesting physics opportunities $[9,10]$.

Luminosities of up to $5 \cdot 10^{31} \mathrm{~cm}^{-2} \mathrm{~s}^{-1}$ can be reached at $\sqrt{s} \simeq 30 \mathrm{GeV}$ in the symmetric $\overline{\mathrm{p}}$ collider mode at the HESR $[11,12]$. The collision scheme of twelve proton bunches colliding with the same amount of antiproton bunches has to be adapted to the HESR. This modification of the HESR requires a second proton injection, the Recuperated Experimental Storage Ring (RESR), the $8 \mathrm{GeV}$ electron cooler and a modification of the PANDA interaction region.

Besides the deceleration of rare-isotope beams, the RESR storage ring also accumulates high-intensity antiprotons, via the longitudinal momentum stacking with a stochastic cooling system [13]. This is achieved by injecting and pre-cooling the produced antiprotons at $3 \mathrm{GeV}$ in the Collector Ring (CR) storage ring.

The antiproton beam intensities in the HESR protonantiproton collider version require a full-energy electron cooler $(8 \mathrm{MeV})$ to avoid beam emittance growth, which results in a decreased luminosity during the cycle. The Budker Institute of Nuclear Physics (BINP) presented a feasibility study for magnetized high-energy electron cooling. An 
electron beam up to $1 \mathrm{~A}$, accelerated in dedicated accelerator columns to energies in the range of $4.5-8 \mathrm{MeV}$ has been proposed. For the FAIR full version, it is planned to install the high-energy electron cooler in one of the HESR straight sections [14-16].

In the fixed-target mode, at $E_{\text {kin }}=4-10 \mathrm{GeV}$, it will be possible to perform complementary measurements of the cross section of charmonium interactions with nuclear matter with the PANDA detector.

A conservative estimate of the $\bar{p}$ luminosities which can be reached at the startup phase without RESR is 4 . $10^{30} \mathrm{~cm}^{-2} \mathrm{~s}^{-1}$. We will use it below in our estimates assuming a one-year run $\left(10^{7} \mathrm{sec}\right)$. Energies of $\sqrt{s}$ up to $30 \mathrm{GeV}$ could be reached and it may also be feasible to study $\bar{p} A$ collisions for $\sqrt{s_{N N}}$ of up to $19 \mathrm{GeV}$. In the present work we outline how the collider at the HESR machine will extend the scope of the PANDA project, with a focus on a few highlights. In Sections 2 and 3 we discuss the potential of the $\mathrm{p} \overline{\mathrm{p}}$ collider to provide new information in the field of heavy quark physics, with some attention devoted to the possible discovery of new states. Some other related opportunities are outlined in Sect. 4. Section 5 presents a number of additional physics topics that could be explored in both $\mathrm{p} \overline{\mathrm{p}}$ and $\overline{\mathrm{p}} A$ modes. These include the production of nuclear fragments containing $\overline{\mathrm{c}} / \overline{\mathrm{b}}$ and/or c/b quarks, $\mathrm{c} \overline{\mathrm{c}} / \mathrm{b} \overline{\mathrm{b}}$ pair production, color-fluctuation effects, probing pure-glue matter, and the production of low-mass dileptons.

Concluding remarks in Sect. 6 close the article.

\section{Study of heavy-quark bound states}

A number of new states containing heavy quarks have been discovered recently. These can be interpreted as pentaquark and tetraquark states containing c $\bar{c}$ pairs. Some of the states are observed in decays of mesons and baryons containing b-quarks, others in the final states of $\mathrm{e}^{+} \mathrm{e}^{-}$annihilation, for a review see [17].

It is widely expected that these discoveries represent just the start of the exploration of rich new families of states containing heavy quarks. Understanding the dynamics responsible for the existence of these states would help to clarify many unresolved issues in the spectroscopy of light hadrons.

A unique feature of an intermediate-energy $\mathrm{p} \overline{\mathrm{p}}$ collider is that it enables the study of the production of $Q \bar{Q}$ ( $Q=\mathrm{c}, \mathrm{b})$ pairs and the formation of various hadrons containing heavy quarks rather close to the threshold. The b $\bar{b}$ pairs are produced mostly by annihilation of valence quarks and antiquarks, i.e. $q \bar{q} \rightarrow Q \bar{Q}$. The production of $\mathrm{c} \overline{\mathrm{c}}$ pairs in the antiproton fragmentation region also corresponds to this mechanism.

The invariant masses of the produced $Q \bar{Q}$ pairs are much closer to the threshold in the discussed energy range than at LHC energies. It is natural to expect that the large probabil- ity to produce final states with small $Q \bar{Q}$ invariant masses should lead to a higher relative probability to produce pentaquark and tetraquark states compared to the one at LHC energies. Additionally, the small transverse momenta of the $Q \bar{Q}$ pairs facilitate the pick up of light quarks as compared to $Q$ or $\bar{Q}$ fragmentation. In the antiproton-fragmentation region another $Q \bar{Q}$ production enhancement mechanism, specific for antiproton interactions, is possible: the production of $Q \bar{Q}$ pairs with large $x \sim 0.2-0.4$ in the annihilation of $q \bar{q}$, which could merge with a spectator antiquark of the antiproton carrying $x \sim 0.2$.

Another effect which can help to observe new states in medium-energy $\bar{p}$ p collisions is the relatively low spatial density of the system produced at moderate energies. This should suppress final-state interactions, which could possibly hinder the formation of weakly bound clusters of large size. An additional advantage is a relatively small bulk hadron production which reduces the combinatorial background significantly as compared to the LHC.

\subsection{The heavy quark production rates}

The need for $\mathrm{t} \overline{\mathrm{t}}$-production cross sections has stimulated the development of new computational techniques for heavyquark production in hadron-hadron collisions (see, e.g., Refs. $[18,19])$, in particular those which include the effects of threshold resummation.

These calculations, which are currently being validated by comparison to data at high collision energies, predict the following cross section for the $b \bar{b}$ pair production in $\bar{p} p$ collisions [20]:

$\sigma_{\mathrm{b} \overline{\mathrm{b}}}(\sqrt{s}=30 \mathrm{GeV})=1.8 \cdot 10^{-2} \mu \mathrm{b}$.

This calculation has a relative uncertainty of about $30 \%$, and the predicted cross-section value is about seven times higher than the corresponding cross section for pp scattering because of the contribution of the valence-quark valence-antiquark annihilation present in $\bar{p}$ p. The $b \bar{b}$ cross section per nucleon for the $15 \mathrm{GeV} \times 6 \mathrm{GeV}$ kinematics is a factor of 100 smaller. Charm production is dominated by gluon annihilation, $\mathrm{gg} \rightarrow$ $Q \bar{Q}$. This fact implies that the corresponding cross sections are similar in $\mathrm{pp}$ and $\mathrm{p} \overline{\mathrm{p}}$ collisions, with the exception $x_{F}$ close to one. The experimental data in this case are rather consistent between pp and $\mathrm{p} p$ and correspond to

$\sigma_{\mathrm{c} \overline{\mathrm{c}}}=30 \mu \mathrm{b}$.

For the $15 \mathrm{GeV} \times 6 \mathrm{GeV}$ scenario the cross section per nucleon drops by a factor of 3 . 


\subsection{Rate estimates}

The cross sections in Eqs. (1) and (2) correspond to significant event rates for 1 year $\left(10^{7} \mathrm{~s}\right)$ of running at a luminosity of $4 \cdot 10^{30} \mathrm{~cm}^{-2} \mathrm{~s}^{-1}$. We find

$N_{\mathrm{b} \overline{\mathrm{b}}}=10^{6}, \quad N_{\mathrm{c} \overline{\mathrm{c}}}=10^{9}$.

These numbers can be easily rescaled for a run at a different luminosity, if required.

In b-mesons and baryons b-quarks carry most of the hadron light cone fraction. In the discussed energy range b-quarks are produced with large light cone fractions $x_{\mathrm{b}} \sim$ $2 m_{\mathrm{b}} / \sqrt{s}$ comparable with those of the valence (anti)quarks, $x_{q}$. However the valence quarks have a large probability to loose their light cone fraction due to the final state interaction. This enhances the coalescence probability of the b-quark and the valence antiquark. One finds a broad distribution over invariant mass of $b \bar{q}$, leading to the expectation of significant formation of excited states in both the meson and the baryon channels, as well as an enhanced probability of the production of the excited $\mathrm{b} \overline{\mathrm{b}} q \bar{q}$ tetraquark or $(\mathrm{b} \bar{q})-(\overline{\mathrm{b}} q)$ mesonic molecular states. The probability for the formation of $b \bar{q}$ mesons with a given flavor is about $30 \%$ of the total cross section because of the competition between different flavors. In the discussed processes gluon radiation is a small correction because of the restricted phase space and the large b-quark mass.

At the collision energies considered here, the combinatorial background is significantly smaller than at the LHC energies, which makes the observation of the excited states containing heavy quarks easier.

\subsection{Hidden beauty resonance production}

In contrast to charm production, the cross section for hidden beauty resonance production $\mathrm{p} \overline{\mathrm{p}} \rightarrow \chi_{\mathrm{b}}$ may be too low to be observed at the HESR. Within the standard quarkonium models, where a $Q \bar{Q}$ pair annihilates into two gluons which subsequently fragment into light quarks, one can estimate that this cross section drops with $M_{Q}$ as $R_{\mathrm{Q}}=\Gamma\left(\chi_{\mathrm{Q}} \rightarrow\right.$ $\mathrm{p} \overline{\mathrm{p}}) / \Gamma_{\text {tot }}\left(\chi_{\mathrm{Q}}\right) \propto \alpha_{s}^{8} / M_{Q}^{8}$. This is because this cross section is proportional to the partial width of the decay $\mathrm{p} \overline{\mathrm{p}} \rightarrow \chi_{\mathrm{b}}$, which drops with an increase of $M_{Q}$. The ratio of cross sections of beauty production through a $\chi_{\mathrm{b}}$ intermediate state to that for charm is $\approx\left[\alpha_{s}\left(M_{Q}\right) / \alpha_{S}\left(M_{c}\right)\right]^{8} /\left[M_{c} / M_{b}\right]^{10}$ with an additional factor of $M_{Q}^{-2}$ stemming from the expression for the resonance cross section. The suppression is due to the necessity of a light-quark rearrangement in the wave functions of the proton and antiproton to obtain decent overlapping with $\chi_{b}$ states. In the non-relativistic approximation the wave functions of $\chi_{b}$ states vanish at zero inter-quark distance. Thus the overall suppression for the total cross section of $\chi_{b}$ production as compared to that of $\chi_{c}$ production is approximately $10^{-7}$.

\section{Potential for discovery of new states}

Investigating $\mathrm{p} \overline{\mathrm{p}}$ collisions at moderate energies carries specific advantages for searches of new states as the $b \bar{b}$ production rate is relatively high, while the overall multiplicity, which determines the background level, is rather modest. Also, an equal number of states containing quarks and antiquarks is produced enabling cross checks of observations using conjugated channels. In the following we will discuss resonances containing heavy quarks with the understanding that everything said equally applies to the resonances containing heavy antiquarks. Although the rates in many cases are rather modest, we nevertheless include the discussion of these channels in view of the possibility to have a higherenergy collider, as discussed in the final remarks (Sect. 6).

\section{$3.1 \mathrm{~b} q q$-baryons and $\mathrm{b} \bar{q}$ mesons}

The current knowledge of the spectrum of the excited states containing $b$ - or $\bar{b}$-quarks is very limited. According to the PDG [21], in the $q \bar{b}$ sector there are two states $\mathrm{B}_{\mathrm{J}}(5970)^{+}$ and $\mathrm{B}_{\mathrm{J}}(5970)^{0}$ with unknown quantum numbers which could be excited states of the $\mathrm{B}^{+}$and $\mathrm{B}^{0}$, respectively. In the bqq-sector there are two baryons $\Lambda_{\mathrm{b}}(5912)^{0}$ and $\Lambda_{\mathrm{b}}(5920)^{0}$ which can be regarded as orbitally excited states of $\Lambda_{\mathrm{b}}^{0}$ and one excited $\Sigma_{\mathrm{b}}^{*}$ state. This is much less in comparison with the c $q q$ sector where five excited $\Lambda_{c}^{+}$states and two excited $\Sigma_{\mathrm{c}}$ states are listed in the Review of Particle Physics (all having a weak experimental rating $* * *)$.

So there are plenty of opportunities here. One noteworthy issue is the comparison of the accuracy with which the heavyquark limit works for hadrons containing b quarks vs. those containing c quarks.

\subsection{Excited states containing $b \bar{b}$}

As argued above it is very difficult to produce bound states containing $b \bar{b}$ in the resonance process of $p \bar{p}$ annihilation. Nevertheless, many of these states, as well as other states like analogs of $X, Y, Z$ charmonium states, could be produced in inelastic $p \bar{p}$ interactions. This is because the invariant mass of the produced $b \bar{b}$ system is rather close to the threshold, and because the $b \bar{b}$ pair is produced in association with several valence quarks and valence antiquarks which have rather low momenta relative to the $\mathrm{b} \bar{b}$ pair. 


\subsection{Baryons and mesons containing two heavy quarks}

Since there are three valence antiquarks colliding with three valence quarks in $\bar{p}$, one can produce two pairs of heavy quarks in a double quark-antiquark collision. This entails a possibility for producing the following baryons and mesons containing two heavy quarks:

(i) $\operatorname{ccq}$ baryons.

At the collision energies discussed, the contribution of the leading-twist mechanism of $2 \mathrm{~g} \rightarrow Q \bar{Q} Q \bar{Q}$ for the double heavy-quark production should be quite small as it requires very large $x$ of the colliding partons (the situation might be less pronounced for the case of the double c $\bar{c}$ production than for $b \bar{b}$ ). Therefore, the only effective mechanism left is the production of two pairs of heavy quarks in two hard parton-parton collisions (see Fig. 1).

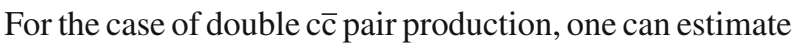
cross sections by considering the suppression factor for the production of the second $c \bar{c}$ pair relative to a single $c \bar{c}$ pair. This factor can be roughly estimated using the high-energy experimental studies of double-parton collisions at the Tevatron collider. One finds a probability of about $10^{-3}$ for the ratio of the cross section for producing two c $\bar{c}$ pairs relative to a single pair.

(To be conservative we took a factor of two larger value of $\sigma_{\text {eff }}=30 \mathrm{mb}$ corresponding to no correlations of gluons in the nucleon wave function. This is a factor of two larger than the value measured at Tevatron where small- $x$ effects reduce $\sigma_{e f f}$, for a recent review see [22].) These considerations result in

$N_{\mathrm{c} \overline{\mathrm{c}}, \mathrm{c} \overline{\mathrm{c}}}=10^{6}$,

as an estimate for the yearly number of events with two pairs of $c \bar{c}$. Since the available phase space is rather modest, there is a significant probability that the relative velocity of two c-quarks would be small, and therefore a ccq state would be formed.

Other interesting channels are the production of an open charm-anticharm pair plus a charmonium, and simultaneous production of two charmoniums.

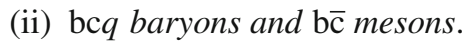

The $b \bar{b} c \bar{c}$ pairs are produced pretty close to threshold and have small relative velocities. Hence there is a good chance that they would form a bc $q$ baryon. About $10^{3}$ events per year of running with $b \bar{b} c \bar{c}$ could be expected based on the double parton interaction mechanism.

(iii) $\mathrm{bb} q$ baryons.

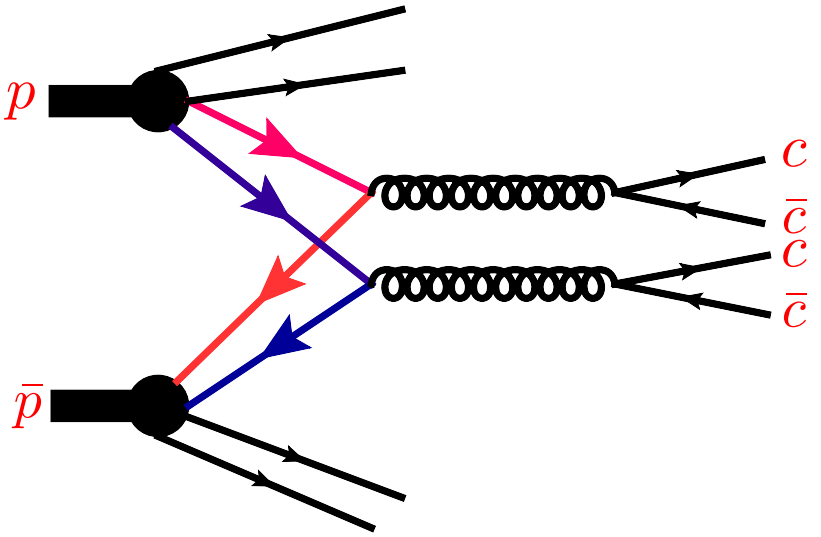

Fig. 2 Double parton interaction mechanism for the production of two pairs of heavy quarks

Assuming bb is produced via double parton interaction mechanism (Fig. 2) we estimate that a production of $\sim 10^{2} \mathrm{~b} \bar{b} b \bar{b}$ would require a a one-year run at a much higher luminosity of $10^{33} \mathrm{~cm}^{-2} \mathrm{~s}^{-1}$. The velocities of two $b$ quarks are expected to be close in about $1 / 2$ of the events. So there should be a significant chance for them to form $\mathrm{bb} q$ baryons.

(iv) bec baryons.

Whether it is feasible to observe bcc states requires more detailed estimates and may depend on the structure of the three-quark configurations in the nucleon. A naive estimate based on the triple parton scattering mechanism is that $10^{2}$ events with bcc $\bar{b} \bar{c} \bar{c}$ would be produced in a one-year run at a higher luminosity of $10^{33} \mathrm{~cm}^{-2} \mathrm{~s}^{-1}$.

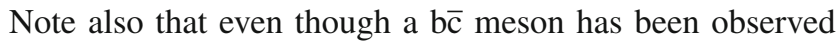
(although its quantum numbers are not known), there are potentially many other states built of these quarks suggesting a rich spectroscopy (mirroring the spectra of $c \bar{c}-$ and $b \bar{b}-$ onium states).

\subsection{Summary}

To summarize, it would be possible with PANDA at the HESR-C $\bar{p} p$ collider to discover and to study properties of meson and baryon states containing one b quark and light (anti) quarks, complementing the charmonium states which are planned to be explored in the PANDA fixed-target experiment. There are also good chances to discover the doubleheavy-quark baryonic and mesonic states. These new potential observations will allow to achieve a much deeper understanding of the bound-state dynamics in QCD. 


\section{Other opportunities}

In the last decades much effort has been devoted to studying high-energy properties of QCD in the vacuum channel - the so-called perturbative Pomeron. Interactions in non-vacuum channels, on the other hand, have practically not been studied. The PANDA experiment at a collider provides a perfect kinematic coverage to study the behavior of Regge trajectories in both the non-perturbative regime (small Mandelstam $|t|$ ) as well as the possible onset of the perturbative regime. One advantage of the antiproton beam is the possibility to study a wide range of baryon and meson Regge trajectories, possibly including Regge trajectories with charmed quarks. The latter will allow to check the non-universality of the slopes of the Regge trajectories which was observed already at positive $t$ values.

Other possibilities include Drell-Yan-pair measurements, which at the lower end of the discussed energy range, may be extended to the limit of exclusive processes like $\bar{p} p \rightarrow$ $\mu^{+} \mu^{-}+$meson which are sensitive to generalized partondistribution functions, etc.

Another direction of studies is the investigation of correlations between valence (anti)quarks in (anti)nucleons using multi-parton interactions (MPI) analogous to those shown in Fig. 2. In the discussed energy range MPI get a significant contribution from collisions of large- $x$ partons (double Drell-Yan, Drell-Yan + charm production, etc.), and the rate of the MPI is inversely proportional to the square of the average distance between the valence quarks. In particular the rates would be strongly enhanced in the case of a large probability of (anti)quark-(anti)diquark configurations in the (anti)nucleon.

The analysis of the production of heavy-quark pairs discussed above would also be of great interest for the study of the multiparton structure of nucleons.

\section{$4.1 \overline{\mathrm{p}}$ and $\overline{\mathrm{p}} A$ elastic scattering and absorption}

Recently the TOTEM collaboration at the LHC performed a high precision measurement of the ratio of the real and imaginary of pp scattering at $\sqrt{s}=13 \mathrm{TeV}$. They found it to be significantly smaller than the expectations based on the vacuum ("Pomeron") C-even exchange[23]. Furthermore, they observed that $\mathrm{pp}$ and $\mathrm{p} \overline{\mathrm{p}}$ elastic cross sections for $\sqrt{s}=1.96 \mathrm{TeV}$ and $\sqrt{s}=2.76 \mathrm{TeV}$, respectively differ strongly in the region of the minimum $\left(-t \sim 0.7 \mathrm{GeV}^{2}\right)$ where the cross section is dominated by the real part of the amplitude [24]. No such significant energy dependence of the real part is expected in the Pomeron + cuts scenario.

This seems to suggest that the contribution of the C-odd amplitude is not as small as suggested by the existing fits based on the $\mathrm{C}$-even dominance. It was suggested to refer to the C-odd exchange as Odderon [25]. In pQCD such an exchange is present as the ladder which starts from the three gluon exchange though its absolute rate cannot be calculated so far; $\mathrm{pQCD}$ model calculations lead to the expectation that the ratio of the Odderon and Pomeron amplitudes should drop with energy. If so, one should look for the Odderon effects at significantly smaller energies than $2 \mathrm{TeV}$. Hence high precision measurements of $\mathrm{Re} / \mathrm{Im}$ in $\mathrm{p} \overline{\mathrm{p}}$ scattering at a range of energies, and their comparison with the pp data would be highly desirable.

A complementary information can be obtained from measurements in elastic antiproton-nucleus collisions. So far such measurements were done only at LEAR for $p_{\text {lab }}<$ $1 \mathrm{GeV} / c$. It has turned out that owing to the forward-peaked $\overline{\mathrm{p}}$ elastic scattering amplitude the Glauber model describes LEAR data on the angular differential cross sections of $\bar{p} A$ elastic scattering surprisingly well. This is in contrast to the $\mathrm{p} A$ elastic scattering where the Glauber model description starts to work only above $p_{\text {lab }} \sim 1.5 \mathrm{GeV} / \mathrm{c}$ [26].

Glauber theory analysis [27] has shown that the $\overline{\mathrm{p}} A$ and $\mathrm{p} A$ angular differential elastic scattering cross sections at $p_{\text {lab }}=10 \mathrm{GeV} / c$ (fixed target PANDA) strongly differ in the diffraction minima due to the different ratios of the realto-imaginary parts of $\overline{\mathrm{p}} N$ and $\mathrm{p} N$ elastic scattering amplitudes. Experimental confirmation of such a behavior would be a good validity test of the Glauber theory, important in view of its broad applications for other reaction channels, and of the input elementary amplitudes which are typically given by Regge-type parameterizations. In particular, the $\overline{\mathrm{p}} \mathrm{n}$ elastic amplitude is accessible only by scattering on complex nuclei. The determination of diffractive structures at $\overline{\mathrm{p}} A$-collider energies would require good transverse momentum transfer resolution $\sim 10 \mathrm{MeV} / c$ and the capability to trigger on the events where the nucleus remains intact (see the discussion in Sect. 5.2). Light nuclear targets are preferred as their diffractive structures are broader in $p_{\mathrm{t}}$, and there is a smaller number of possible excited states. Spin-0 targets like ${ }^{4} \mathrm{He}$ are especially suitable for these purposes. Since ${ }^{4} \mathrm{He}$ has zero spin the depth of the minimum at $-t \approx 0.2 \mathrm{GeV}^{2}[28]$ is given by the real part of the amplitude. Note that in this case the contribution of the Reggeon-exchange like $\rho$ exchange is absent due to the zero isospin of the deuteron.

A related problem is the determination of the antiproton absorption cross section on nuclei (defined as the difference between total and elastic cross sections). Experimental data on the antiproton absorption cross section above LEAR energies are quite scarce, although such data are needed for cosmic ray antiproton flux calculations [29].

\subsection{Rapidity migration of the fragments of the projectile}

The dynamics of the fragmentation of the hadronic projectiles in the scattering off protons and nuclei is not studied in much detail especially for forward rapidities. The use of 
antiprotons for these purposes has certain advantages as compared to the use of protons as for large rapidity intervals it allows to distinguish contributions of the projectile and nucleus fragmentation. It would be possible to study how much the fragmentation of the projectile is suppressed as a function of multiplicity of hadrons produced at the central rapidities as well as in the nucleus fragmentation region. Use of several nuclei would allow to study the dependence of the rapidity distribution of $\overline{\mathrm{p}}, \bar{\Lambda}$, etc. as a function of the nuclear thickness, $T(b) \int_{-\infty}^{\infty} \mathrm{d} z \rho_{A}(b, z)$. In particular one would be able to study how the spectra of antibaryons having one or two common antiquarks vary with the atomic number of the projectile.

\subsection{Coherent hypernuclei production}

While ordinary $\Lambda$-hypernuclei were discovered long ago, the $\Lambda_{\mathrm{c}}^{+}$- and $\Lambda_{\mathrm{b}}^{0}$-hypernuclei were predicted in the mid-70s $[30,31]$ but have not been observed so far. However, their existence is expected based on a number of models, e.g. the quark-meson coupling model [32].

The processes $\overline{\mathrm{p}} \mathrm{p} \rightarrow \bar{Y} Y$, where $Y=\Lambda, \Lambda_{\mathrm{c}}^{+}$or $\Lambda_{\mathrm{b}}^{0}$, have the lowest thresholds among all possible other channels of the respective $\bar{s} s, \bar{c} c$ or $\bar{b} b$ production channels in $\bar{p} p$ collisions. Thus, they are preferred for $Y$-hypernuclei production as the momentum transfer to the hyperon is relatively small.

The coherent reactions ${ }^{A} Z(\overline{\mathrm{p}}, \bar{\Lambda}){ }_{\Lambda}^{A}(Z-1)$ for the different states of the hypernucleus have never being studied experimentally. It is expected that these reactions have cross sections of the order of a few $10 \mathrm{nb}$ at $p_{\text {lab }} \sim 20 \mathrm{GeV} / c[33]$. Thus, they can serve as a powerful source of $\Lambda$-hypernuclei production at the lower end of the $\overline{\mathrm{p}} A$-collider energies. Here, the amplitude $\overline{\mathrm{p}} \mathrm{p} \rightarrow \bar{\Lambda} \Lambda$ should be dominated by the $\mathrm{K}^{*}$ (or $\mathrm{K}^{*}$ Regge trajectory) exchange.

More challenging is the coherent process ${ }^{A} Z\left(\overline{\mathrm{p}}, \bar{\Lambda}_{\mathrm{c}}^{-}\right){ }_{\Lambda_{\mathrm{c}}^{+}}^{A} Z$ [34] where the underlying $\overline{\mathrm{p} p} \rightarrow \bar{\Lambda}_{\mathrm{c}}^{-} \Lambda_{\mathrm{c}}^{+}$amplitude is due to $\mathrm{D}^{0}$ and $\mathrm{D}^{* 0}$ exchanges. One can also think of the ${ }^{A} Z\left(\overline{\mathrm{p}}, \bar{\Lambda}_{\mathrm{b}}^{0}\right){ }_{\Lambda_{\mathrm{b}}^{0}}^{A}(Z-1)$ coherent reaction.

\section{Unique opportunities for probing QCD properties at the $\overline{\mathbf{p}} A$ collider}

\subsection{Space-time picture of the formation of hadrons} containing heavy quarks

The kinematics of heavy-quark-state production in collisions of $\mathrm{p}(\overline{\mathrm{p}})$ with proton or nuclei (neglecting Fermi motion effects) dictates that heavy states can only be produced with momenta

$$
p_{Q}>M_{Q}^{2} / 2 m_{N} x_{q}-m_{N} x_{q} / 2
$$

in the rest frame of the nucleus. Here $x_{q}$ is the $x$ of the quark of the nucleus involved in the production of the $Q \bar{Q}$ pair. For $x_{q} \leq 0.5$ this corresponds to a charm momentum above $4 \mathrm{GeV} / c$ which is much larger than typical momenta of the heavy system embedded in the nucleus.

However, there is a significant probability that $\mathrm{D}, \Lambda_{\mathrm{c}}, \ldots$ hadrons slow down due to final-state interactions. Indeed, it is expected in QCD that the interaction strength of a fast hadron with nucleons is determined by the area in which the color is localized. For example, the cross section of the $\psi^{\prime}$ $N$ and kaon-nucleon interaction should be comparable. At the same time the $\psi^{\prime}-N$ cross section should be much larger than the $\mathrm{J} / \psi-N$ cross section, since the color is localized in a small volume in $J / \psi$, see for example discussion in [35]. Also the cross sections of open charm (bottom) interactions should be on the scale $\gtrsim 10 \mathrm{mb}$.

The formation distance (coherence length) can be estimated as

$l_{\mathrm{coh}} \simeq \gamma l_{0}$

where $l_{0} \simeq 0.5-1.0 \mathrm{fm}$. For the discussed energies and the case of scattering off heavy nuclei the condition

$l_{\text {coh }} \leq R_{A}$,

is satisfied for hadrons produced in a broad range of momenta including the central and nucleus fragmentation region. So it would be possible to explore the dependence of the formation time and interaction strength on, for example, the orbital angular momentum of $\mathrm{D}^{*}$. This would allow to test the expression for the formation distance $l_{\mathrm{coh}}=2 p / \Delta M^{2}$ [36], where $p$ is the hadron momentum and $\Delta M^{2}$ is typically given by the difference of masses of the hadron and the closest mass states with the same quantum numbers.

Observing these phenomena and hence exploring QCD dynamics in a new domain could be achieved by studying the $A$-dependence of charm production at momenta $\lesssim 10 \mathrm{GeV} / c$ (in the rest frame of the nucleus).

\subsection{Charm bound to nuclear fragments}

The formation of heavy mesons or baryons, $\mathrm{H}$, inside nuclei implies final-state interactions which slow down these heavy hadrons, leading to the production of hadrons at low momenta forbidden for scattering off a free proton (cf. Eq. 5):

$p_{\mathrm{H}} \leq\left(m_{\mathrm{H}}^{2}-m_{N}^{2}\right) / 2 m_{N}$.

In this kinematics the slow-down may be sufficient to allow for the production of (anti-)charm quarks embedded in nuclear fragments. The collider kinematics would make it easier to detect decays of such nuclei than in fixed-target 
setups as these nuclei would be produced with high momenta (velocities comparable to those of ordinary nuclear fragments). Thus the discussed HESR-C collider in the $\overline{\mathrm{p}} A$ mode would have a high discovery potential for observing various nuclear states containing $\mathrm{c}$ and/or $\overline{\mathrm{c}}$.

Higher luminosities and higher collider energies will allow the search for analogous $b$ and/or $\bar{b}$ states.

\subsection{Color fluctuations in nucleons}

At high energies hadrons are thought to be interacting with each other in frozen configurations which have different interaction strengths - so-called color fluctuations. One can explore these phenomena in (anti)proton-nucleus collisions in a number of ways. Here we give as one example the study of the interaction strength of a hadron in the case of a configuration that contains a large- $x(x \geq 0.4)$ parton. One expects that in such configurations the average interaction strength is significantly smaller than $\sigma_{\text {tot }}(h N)$. In these configurations color screening leads to a suppression of the gluon fields and of the quark-antiquark sea [37]. This picture has allowed to explain $[38,39]$ strong deviations of the centrality dependence of the leading-jet production from the geometrical picture (Glauber model of inelastic collisions) observed at the LHC in $\mathrm{p}-\mathrm{Pb}$ collisions and at RHIC in d-Au collisions.

Due to a fast increase of the interaction strength for smallsize configurations with increasing energy, the strength of color fluctuations drops at higher energies. Correspondingly color-fluctuation effects are expected to be much enhanced at the HESR-C $\overline{\mathrm{p}} A$-collider energies. For example, for $x \simeq$ 0.6 , the cross-section ratio, of the average strength of the interaction of a proton in a configuration containing a quark with given $\mathrm{x}, \sigma_{\text {eff }}(x)$ and $\sigma_{\text {tot }}(N N)$, is expected to be $\sim 0.25$, while at the $\mathrm{LHC}$ it is $\sim 0.6$.

To observe this effect one would need to study Drell-Yan production at large $x$. A strong reduction of hadron production in the nucleus fragmentation region would be a strong signal for the discussed effect. For its detailed study measurements with different nuclei would be desirable.

\subsection{Study of short range correlations at a hadron collider}

\subsubsection{Brief summary of the studies of the short-range correlations}

The presence of the high momentum correlated component in the nuclear wave function was expected within microscopic theories of nuclei since the 1960s, see for example [40-42].

A distinctive feature of these correlations is that the singular nature of the short range $\mathrm{NN}$ interaction generates a universal (up to normalization and isospin effects) highmomentum component in the nucleus wave function with the fast nucleon momentum balanced by a few nucleons, pre- dominantly by one $-2 N$ short range correlation (SRC). In the SRC the distances between nucleons are much smaller than on average, leading to local densities comparable to densities in the cores of neutron stars.

Thus the investigation of the inner structure of SRCs and the pattern of balancing the large momentum recoil are an important step in probing the dynamics of the cores of neutron stars in the laboratory.

A number of attempts to observe experimentally such correlations have been undertaken and it has been repeatedly found that multistep processes mask the contribution of the SRCs leading to a widely held belief that SRCs cannot be observed. However, the multistep processes in similar reactions may probe the cumulative phenomenon of subthreshold particle production [43]. This has been confirmed by microscopic transport calculations $[44,45]$ and is still to be verified experimentally.

It has been concluded in $[46,47]$ that these failures are explained by the lack of a significant difference between the scales of the energy-momentum of the probed bound state and the energy-momentum transfer in the measured process.

The condition for resolving said $2 N \mathrm{SRCs}$ is that the energy- and momentum transfer, $q$, satisfies the condition

$q_{0} \gg k^{2} / m_{N}, \quad q_{3} \gg 2 k$,

where $k$ is the momentum of struck nucleon.

The first high-energy signal for the presence of SRCs in nuclei comes from the studies of the processes

$a+A \rightarrow$ fast backward nucleon $+\mathrm{X}, a=p, \pi, \gamma, \nu$,

in the kinematics where the Feynman $x_{\mathrm{F}}$ for the nucleon scaled to A exceeds $1+k_{\mathrm{F}} / m_{N}$, where $k_{\mathrm{F}}$ is the nucleon Fermi momentum.

We will discuss the case of the fragmenting nucleus viewed as a "projectile". In the frame where the nucleus is fast, the Feynman variable can be thus defined as

$x_{\mathrm{F}}=\frac{p_{N}^{z}}{P_{A}^{z}}$,

where $p_{N}^{z}$ and $P_{A}^{z}$ are the longitudinal momenta of the emitted nucleon and the nucleus, respectively. In the case of a hadron-nucleus interaction it is convenient to introduce a scaled variable

$\alpha_{N}=x_{\mathrm{F}} A$.

In the reference frame, where the nucleus has large a momentum $P$,

$\alpha_{N}=A p_{N} / P$ 
Since the produced nucleon cannot carry the momentum fraction larger than 1 of the involved nucleon subsystem, the production of a nucleon with $\alpha \geq j$ (where $j$ is an integer $\geq 1$ ) requires a process involving at least $j+1$ nucleons.

The spectator mechanism of the production of a fast backward nucleon has been proposed in which the projectile hadron (photon, neutrino) interacts inelastically with one nucleon of the $2 N$ SRC, carrying a light-cone fraction $\alpha_{1}$ and releasing the second nucleon of the SRC [46] with $\alpha_{2} \approx 2-\alpha_{1}$ (Note here that in typical high energy interactions with a nucleon and momentum transfer to the nucleon satisfy Eq. 9). The model predicts that the spectra of nucleons from deuteron and ${ }^{4} \mathrm{He}$ would be close between $\alpha \geq 1.3$ and $\alpha \sim 1.6$ with an upper limit determined by the contribution from $3 N$ SRCs and that for $A \geq 4$ the $\alpha$-distributions are practically the same at least up to $\alpha \sim 2$. These expectations have been confirmed by the data; for a summary see [47].

Furthermore for light nuclei the relative probability of $2 \mathrm{~N}$ correlations in the nuclei and the deuteron has been determined which has been confirmed in large- $Q^{2}, x \geq 1.4$ inclusive $A\left(\mathrm{e}, \mathrm{e}^{\prime}\right)$ reactions [48] which have tested both the universality of $2 N$ correlations and the dominance of the light-cone dynamics in the (e,e') reactions.

The following studies have focused on quasi exclusive reactions starting with the large momentum transfer $(-t \sim$ $5 \mathrm{GeV}^{2}$ ) reaction

$\mathrm{p}+\mathrm{C} \rightarrow \mathrm{pp}+$ backward neutron $+(A-2)^{*}$.

It has been observed that for momenta $\geq 250 \mathrm{MeV}$ of the struck nucleon in about $90 \%$ of the scattering events of a fast proton a backward neutron with balancing momentum is produced [49]. Such a dominance of neutron emission is the natural consequence of the dominance of $I=0, S=1 \mathrm{NN}$ SRCs in the nucleus wave function.

Further studies at Jlab of the reactions

$\mathrm{e}+A \rightarrow \mathrm{e}+$ forward $\mathrm{p}+$ spectator $\mathrm{n}(\mathrm{p})+(A-2)^{*}$,

$e+A \rightarrow \mathrm{e}+$ forward $\mathrm{n}+$ spectator $\mathrm{p}+(A-2)^{*}$,

at $x \geq 1.2, Q^{2} \geq 1.5 \mathrm{GeV}^{2}$ have confirmed the dominance of the scattering off $2 \mathrm{~N}$ SRCs and tested the dominance of the pn SRCs (see [50] and references therein). Note that measurements with proton and electron projectiles are complementary as in the case of the proton projectile the primary interaction occurs with the forward nucleon, and the spectator is emitted in the backward direction, while in the electron case the kinematics has been studied in which the electron is interacting with a backward nucleon $(\alpha>1)$ and the spectator is emitted forward. Also, due to a stronger absorption in the case of proton projectiles, the proton induced reactions probe lower densities than electron-induced processes. The collider kinematics has a number of advantages as compared to the fixed target setup. In particular, in the Collider setup it is rather easy to detect all products of interactions with a SRC - as they move along the projectile direction. On the contrary, in case of the proton projectile the SRC constituents fly in different directions - both forward and backward.

Comment. The concept of the relativistic nucleon momenta within a SRC is beyond the framework of the non-relativistic theory. Popular model assumptions like the ones used in $[51,52]$ violate probability conservation as well as the global symmetries. They also lead to the presence of certain configurations in nuclei which cannot be knocked out from the nucleus - an obvious contradiction with quantum mechanics. In the relativistic limit one has to take into account the production of virtual $N \bar{N}$ pairs. These effects can be adequately taken into account in light-cone quantum mechanics of nuclei which is in a direct correspondence with the spacetime evolution of high-energy processes (for an extensive discussion see [47]). Note also that high-energy processes allow to investigate modifications of quark and gluon distributions in bound nucleons and the onset of the transition to collective behavior of quarks and gluons which is manifested in the EMC effect.

\subsubsection{Opportunities at Colliders}

In the collider kinematics it would be possible to study in greater detail the dynamics of interactions of antiprotons with $2 \mathrm{~N}$ correlations in the inelastic channels including shadowing due to simultaneous interaction with two nucleons of the correlation, studying transverse momentum balance between an $\alpha_{N}<1$ nucleon (fast backward in the nucleus rest frame) and $\alpha_{N}>1$ ) nucleons (forward-emitted in the nucleus rest frame). The selection of events with fast backward nucleons may give unique information about the interplay between Glauber like mechanisms of knock out of nucleons and mechanisms involving secondary cascade interactions.

It would also be possible to study quasi-elastic channels for a range of momentum transfers above $1 \mathrm{GeV}^{2}$ for the scattering off both $\alpha_{N}>1$ and $\alpha_{N}<1$ nucleons (in the case of medium energies only studies of scattering off $\alpha_{N}<1$ moving nucleons is feasible due to the strong suppression of the cross section for scattering at large angles).

Using hadronic projectiles may play a very important role in the studies of higher order (in particular 3N) SRCs. Such correlations can emerge from an iteration of two hard $N N$ interactions or from the genuine three-nucleon interaction. The ratio of the probability of $3 N$ and $2 N$ SRCs is expected to grow with nuclear density for large nucleon momenta. Hence they could play a significant role in the cores of the neutron stars.

The current evidence for the $3 N$ SRCs comes from the studies of the reaction (10) for $\alpha \geq 1.6$ where contribution of $2 N$ SRCs is suppressed, from modeling $3 N$ SRCs as an 
iteration of the hard interactions of two nucleons [47], studies of the ${ }^{3} \mathrm{He}$ wave function [53], as well as studies of the (e,e') reaction at $x \geq 2.5$ [54].

The studied reactions do not explicitly probe $3 N$ correlations as they do not measure the final state ((e,e') reactions) or just measure one nucleon (reaction 10). Obviously more exclusive measurements are necessary. The production dynamics of a nucleon spectator from $j>2$ SRCs is more complicated than in the case of $2 N$ SRCs. In the $2 N$ case it is sufficient to transfer a large energy and momentum to the second nucleon (9). However in the case of $3 N$ SRCs this is not sufficient, as the second nucleon of the correlation does not receive a large kick, and the bond between this nucleon and the spectator is not destroyed, leading to a significant final-state interaction and a suppression of the yield of the fast nucleon. A much more effective mechanism is the interaction of the projectile with two nucleons balancing a large $\alpha$ nucleon [47]. In the case of hadronic projectiles such double interactions occur without suppression since the three nucleons are close to each other. However in DIS such interactions are strongly suppressed (if $x$ is not small). Hence a comparison of the $\mathrm{p}(\overline{\mathrm{p}}) A$ and $\gamma^{*} A$ production of fast backward nucleons would allow to separate different SRCs. It would be also possible to use the final-state information to select events in which one or two nucleons of the target are involved in the high-energy interactions with the projectile. In the case of quasielastic scattering at large $|t|$ one can also separate events originating from $2 N$ and $3 N$ SRCs by studying how many nucleons are balancing the nucleon with large $\alpha$ and $p_{t}^{2} \geq 2 \mathrm{GeV}^{2}$. In the case of two nucleons it should be predominantly a pn system, for three nucleons - ppn or nnp: $h+A \rightarrow h\left(p_{t} \geq 1.5 \mathrm{GeV}\right)+\mathrm{pn}+(A-2)^{*}$, $h+A \rightarrow h\left(p_{t} \geq 1.5 \mathrm{GeV}\right)+\operatorname{ppn}(\mathrm{nnp})+(A-2)^{*}$.

At the collider one can also study effects of nonnucleonic degrees of freedom like $\Delta$ isobars, $N^{*}$, etc.

Obviously these would be challenging measurements, with their interpretations leading to a better understanding of the dynamics of hadron-nucleus scattering. Having complementary programs at fixed-target and electron-ion colliders would be critical for the success of such studies as comparisons of these seemingly different reactions would test the factorization of observed cross sections into a product of the elementary cross section and nuclear decay function and address the question of the role of the f.s.i. which are very different in the electron and hadron reactions.

\subsection{Probing pure glue matter}

One of the central questions in high-energy hadronic and nuclear collisions is how the initially non-equilibrium system evolves towards a state of apparent (partial) thermodynamic equilibrium at later stages of nuclear collisions. Presently, the community favors a paradigm of an extremely rapid (equili- bration time $t_{\mathrm{eq}}$ less than $0.3 \mathrm{fm} / c$ ) thermalization and chemical saturation of soft gluons and light quarks.

The large gluon-gluon cross sections lead to the idea [55] that the gluonic components of colliding nucleons interact more strongly than the quark-antiquark ones. A two-step equilibration scenario of the quark-gluon plasma (QGP) has been proposed in [56-58]. It has been assumed that the gluon thermalization takes place at the proper time $\tau_{\mathrm{g}}<1 \mathrm{fm} / c$ and the (anti)quark equilibration occurs at $\tau_{\mathrm{th}}>\tau_{\mathrm{g}}$. The estimates of Refs. [59-61] show that the quark equilibration time $\tau_{\text {th }}$ can be of the order of $5 \mathrm{fm} / c$. More recent studies [62,63], based on QCD effective kinetic theory [64], suggest smaller values of $\tau_{\mathrm{th}} \sim 1-2 \mathrm{fm} / c$ and that a longitudinally expanding system is not yet thermalized at chemical equilibration but exhibits a considerable momentum anisotropy. The hydrodynamic behavior, however, sets in before chemical equilibration, making a viscous fluid dynamical description applicable already at the early stages $[65,66]$. In the present exploratory study we shall focus on the effects of chemical non-equilibrium while preserving the ideal hydrodynamic description for simplicity. A more quantitative future study shall take into account the viscous corrections and effects of momentum anisotropy.

The pure glue scenario has been proposed for the initial state at midrapidity in $\mathrm{Pb}+\mathrm{Pb}$ collisions at the Relativistic Heavy Ion Collider (RHIC) and Large Hadron Collider (LHC) energies [67,68]. According to lattice-QCD calculations [69], quarkless purely gluonic matter should undergo a first-order phase transition at a critical temperature $T_{\mathrm{c}}=270 \mathrm{MeV}$. At this temperature the deconfined pure-glue matter transforms into the confined state of pure Yang-Mills theory, namely into a glueball fluid. This is in stark contrast to full QCD equilibrium with $(2+1)$ flavors, where a smooth crossover transition takes place (see Fig. 3 for a comparison of the corresponding equations of state).

At $\sqrt{s_{N N}} \simeq 30 \mathrm{GeV} \overline{\mathrm{p}}(A)$ collisions can create only small systems. Baryon free matter can be expected if the $\bar{p} p$ annihilation occurs briefly in the initial stage of the collision. An enhanced annihilation probability can be expected in $\overline{\mathrm{p}} A$ collisions over the $\bar{p}$ p collisions. However, the matter created in $\overline{\mathrm{p}} A$ collisions will not be net-baryon free. In the following we focus on the particular class of $\bar{p} p$ events where baryon free matter is created. Note that the described $\bar{p} p$ collisions at PANDA are quite different from the heavy-ion program of the future compressed baryonic matter (CBM) experiment at FAIR. The heavy-ion collisions at CBM will create excited baryon-rich QCD matter rather than baryon-free pure glue matter in PANDA. Therefore, the PANDA program will be complementary to the CBM experiment.

If indeed a hot thermalized gluon fluid, initially containing no (anti)quarks, is created in the early stage of a $\bar{p} p$ (or $\overline{\mathrm{p}} A$ collision) at mid rapidity, it will quickly cool and expand until it reaches a mixed-phase region at $T=T_{\mathrm{c}}^{\mathrm{YM}}$. After the 


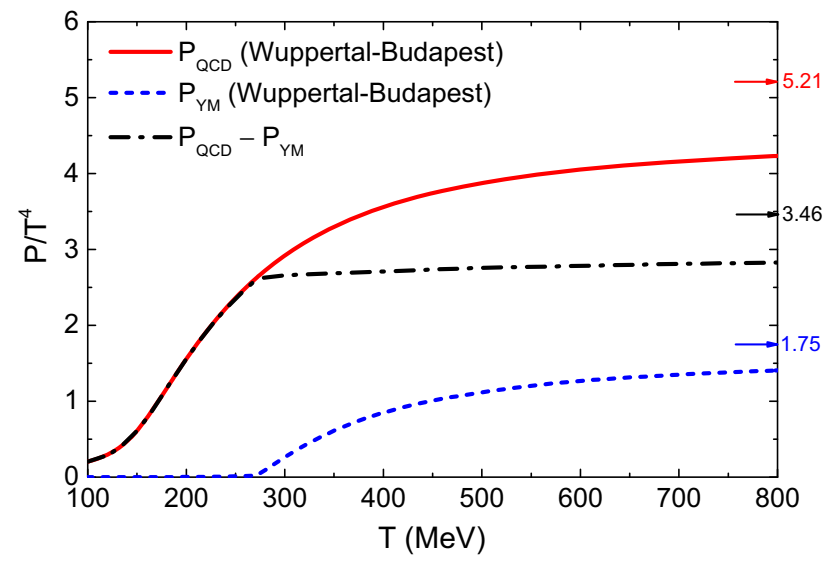

Fig. 3 Temperature dependence of the scaled pressure, $p / T^{4}$, obtained in lattice QCD calculations of the Wuppertal-Budapest collaboration for $(2+1)$-flavor QCD [75] (red line) and for Yang-Mills matter [69] (blue line). The black dash-dotted line depicts the difference between the pressure in full QCD and in Yang-Mills theory

initial pure gluon plasma has completely transformed into the glueball fluid, the system will cool down further. The heavy glueballs produced during the Yang-Mills hadronization process, where the pure glue plasma forms a glueball fluid, will later evolve into lighter states, possibly via a chain of twobody decays [70], and finally decay into hadronic resonances and light hadrons, which may or may not show features of chemical equilibration.

Of course, a more realistic scenario must take into account that some quarks will be produced already before and during the Yang-Mills driven first-order phase transition. This scenario can be modeled by introducing a time-dependent effective number of (anti)quark degrees of freedom, given by the time-dependent absolute quark fugacity $\lambda_{q}$ [71]:

$\lambda_{q}(\tau)=1-\exp \left(\frac{\tau_{0}-\tau}{\tau_{*}}\right)$

Here $\tau_{*}$ characterizes the quark chemical equilibration time, and $\tau$ is the longitudinal proper time.

To illustrate the above considerations, we apply the $(2+$ 1)-dimensional relativistic hydrodynamics framework with a time-dependent equation of state, developed in Refs. [72, 73] and implemented it in the vHLLE package [74], to $p \bar{p}$ collisions at HESR. The equation of state interpolates linearly between the lattice equations of state for the purely gluonic Yang-Mills (YM) theory [69] $P_{\mathrm{YM}}(T)$ at $\lambda_{q}=0$ and for the full QCD with $(2+1)$ quark flavors [75] $P_{\mathrm{QCD}}(T)$ at $\lambda_{q}=1$ :

$$
\begin{aligned}
P\left(T, \lambda_{q}\right) & =\lambda_{q} P_{\mathrm{QCD}}(T)+\left(1-\lambda_{q}\right) P_{\mathrm{YM}}(T) \\
& =P_{\mathrm{YM}}(T)+\lambda_{q}\left[P_{\mathrm{QCD}}(T)-P_{\mathrm{YM}}(T)\right] .
\end{aligned}
$$

$P_{\mathrm{YM}}(T)$ and $P_{\mathrm{QCD}}(T)$ are shown in Fig. 3.
The hydrodynamic simulations of $\mathrm{p} \overline{\mathrm{p}}$ collisions at $\sqrt{s}=$ $30 \mathrm{GeV}$ discussed below assume a hard-sphere initial energy density profile with radius $R=0.6 \mathrm{fm}$. The normalization of the energy is fixed in order to yield an initial temperature of $273 \mathrm{MeV}$ in the central cell, which is slightly above the critical temperature of $270 \mathrm{MeV}$. This choice is motivated by Bjorken-model based estimates at $\sqrt{s}=30 \mathrm{GeV}$ for small systems [76].

Figure 4a shows the $\tau$-dependence of the temperature in the central cell for different quark equilibration times: $\tau_{*}=0$ (instant equilibration), $1 \mathrm{fm} / c$ (fast equilibration), $5 \mathrm{fm} / c$ (moderate equilibration), $10 \mathrm{fm} / c$ (slow equilibration), and $\tau_{*} \rightarrow \infty$ (pure gluodynamic evolution). In the pure gluodynamic scenario, $\tau_{*} \rightarrow \infty$, the system spends a very long time in the mixed-phase region. A fast quark equilibration shortens the time period spent in the mixed phase significantly. Nevertheless, a significant fraction of the system evolution takes place in the mixed phase of the gluonglueball deconfinement phase transition for a moderately fast quark equilibration ( $\tau_{*}=5 \mathrm{fm} / c$ ), as illustrated by Fig. $4 \mathrm{~b}$. Thus, significant effects of the initial pure-glue state on electromagnetic and hadronic observables are expected for this collision setup.

These results illuminate the future HESR-collider option with the central PANDA experiment detector as an exciting upgrade for FAIR, a promising option to search for even heavier glueballs and hadrons than envisioned for the fixed target mode, and for other new exotic states of matter. Of course, the results have been obtained within a largely schematic calculation of $\bar{p}$ collision dynamics which employs ideal hydrodynamics and chemical equilibration of quarks put in by hand. Nevertheless, we hope that the possibility to observe remnants of the first-order phase transition in pure gluodynamics suggested by the result will motivate experimental measurements at PANDA as well as more detailed theoretical studies based e.g. on viscous hydrodynamics or partonic transport theory.

\subsection{Constituent antiquark-quark Drell-Yan processes and dilepton production}

Low-mass lepton pair production has raised the interest in the field for decades. A quite robust theoretical understanding [77-82] of dilepton production in heavy-ion collisions at various energies has been gained. There dileptons play a special role as messengers from the early stages, as penetrating probes.

At large invariant dilepton masses the perturbative DrellYan (DY) mechanism of QCD sets in. The minimal $M_{\mathrm{DY}}^{2}$ value, where the DY mechanism dominates, is not well known as it is difficult to separate it experimentally from the contribution of charm and $\mathrm{J} / \psi$ production. It seems that the DY pair production mechanism dominates at $M_{\mathrm{DY}} \geq 2 \mathrm{GeV}$. 


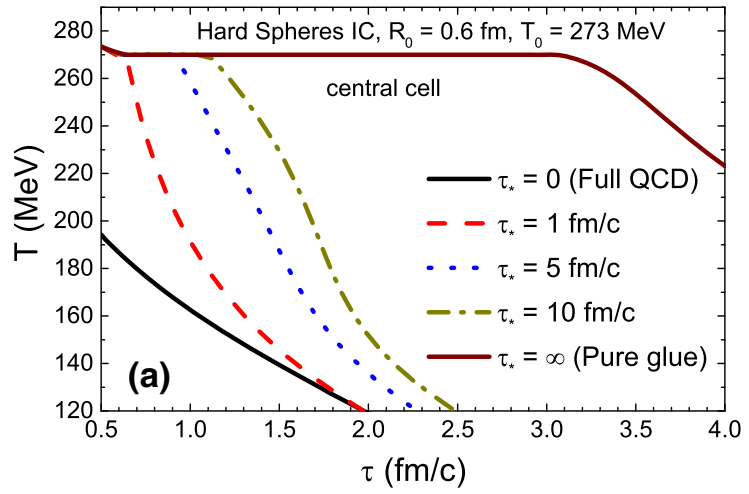

Fig. 4 The temperature profile of the central cell in the longitudinally boost invariant $(2+1)$-dimensional hydro evolution for $\mathrm{p} \overline{\mathrm{p}}$ collisions in the pure-glue initial state, the Yang-Mills scenario. A hard spheres overlap, transverse density profile with radius $R=0.6 \mathrm{fm}$ is used as the initial condition. The normalization is fixed in order to yield an initial temperature of $273 \mathrm{MeV}$ in the central cell. a The $\tau$-dependence of the

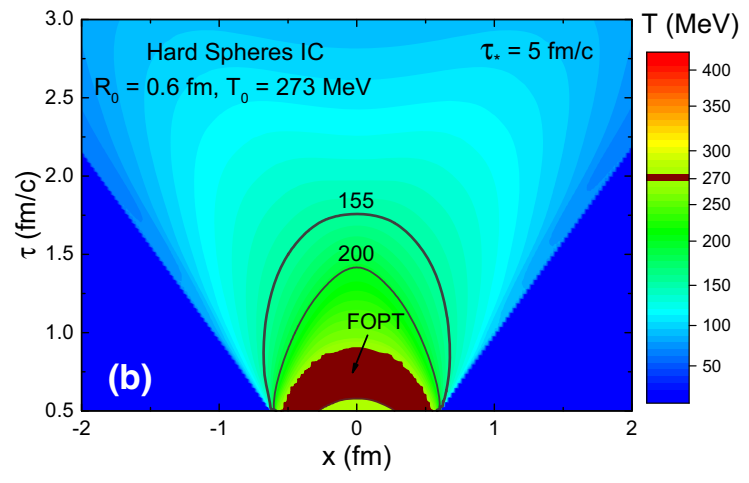

temperature is given for the central cell for different quark equilibration times: $\tau_{*}=0$ (instant equilibration), $1 \mathrm{fm} / c$ (fast equilibration), $5 \mathrm{fm} / c$ (moderate equilibration), $10 \mathrm{fm} / c$ (slow equilibration), and for $\tau_{*} \rightarrow \infty$ (pure gluodynamic evolution). $\mathbf{b}$ The temperature profile in the $x-\tau$ plane for $\tau_{*}=5 \mathrm{fm} / c$

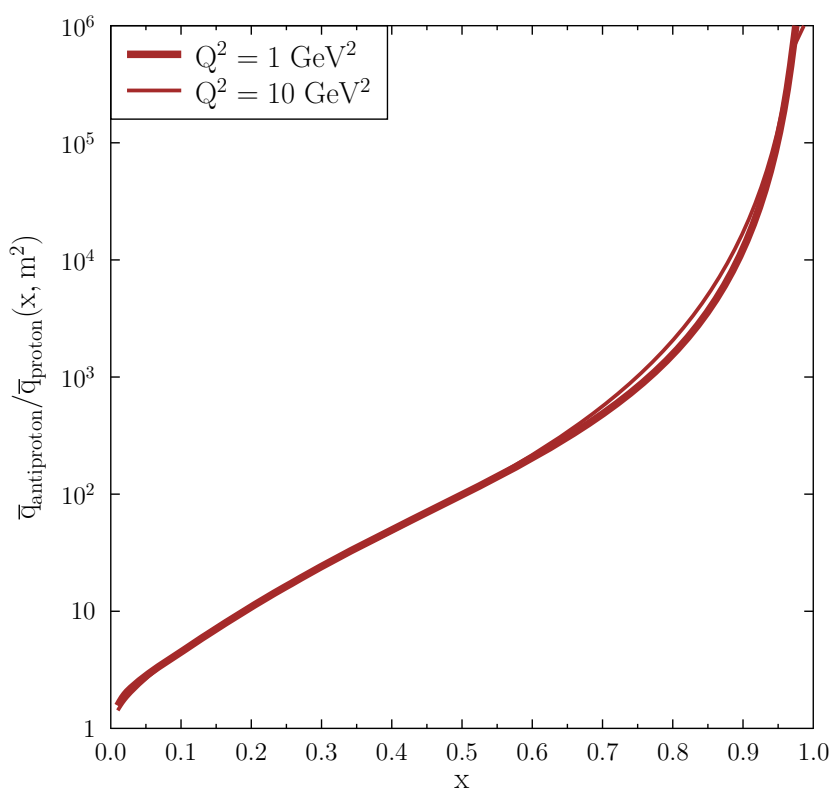

Fig. 5 Left: The GRV LO light-anti-quark parton-distribution functions (PDFs) at $m^{2}=10 \mathrm{GeV}^{2}$ (thin lines) and $m^{2}=1 \mathrm{GeV}$ (bold lines) for antiprotons, protons [83], and pions [84]. Right: The ratio of the PDFs for light anti-quarks in antiprotons and in protons. For further details, see [85-87]

For $\overline{\mathrm{p}} \mathrm{p}(A)$ collisions at intermediate energies, $M_{\mathrm{DY}}$ should be lower than for proton projectiles due to the lower background contributions to lepton pairs from hadronic decay because of the presence of abundant valence antiquarks as discussed above.

Higher-twist mechanisms may delay the inset of the leading-twist contribution in the case of interactions with nuclei. Drell-Yan production of dileptons at intermediate invariant masses by reactions with secondary mesons and (anti-)baryons in $\mathrm{p} A$ and $A A$ collisions has been investigated in [85-87]. At the lower beam energies of the beam- energy scan at RHIC, at FAIR, and at NICA, strong contributions from secondary DY processes are predicted for lowand intermediate-mass dilepton pairs due to the formation of mesons at time scales $\lesssim 1 \mathrm{fm} / c$, i.e. during the interpenetration stage of projectile and target. This implies that inflying primordial projectile and target nucleons from the interpenetrating nuclei collide with just newly formed mesons (e.g., $\rho$ and $\omega$ with constituent-quark and constituent-antiquark masses of $\sim 300 \mathrm{MeV}$ each).

For $\overline{\mathrm{p} p}$ and $\overline{\mathrm{p}} A$ reactions, the Drell-Yan production is enhanced already for the primordial collisions due to the 
presence of valence antiquarks of the antiproton, as displayed in Fig. 5 which shows the ratio of light-antiquark PDFs in antiprotons and protons. With the HESR-C $\bar{p} p$ and $\overline{\mathrm{p}} A$ collider discussed here a direct assessment of the valence-quark valence-antiquark parton distributions in $\overline{\mathrm{p}} \mathrm{p}$ and $\overline{\mathrm{p}} A$ collisions is accessible. The comparison of dilepton production by proton and antiproton induced reactions provides unique information on the relative role of initial- and final-state mechanisms. In particular, by comparing dilepton production in proton and antiproton fragmentation regions one may expect the maximal difference between the two cases.

Calculations of the inclusive (integrated over transverse momenta) DY production can be performed now in the NNLO DGLAP approximation. The techniques were developed for calculations of transverse momentum distributions which include effects of multiple gluon emissions (the Sudakov form factor effects) and nonperturbative transverse momentum distributions (TMD), see e.g. [88] and references therein. TMDs contribute to the differential cross section predominantly at small transverse momenta of the DY pairs and for moderate masses of the pairs.

For large masses the theory works now very well providing a parameter free description of $Z$-boson production at the LHC including the transverse-momentum distribution.

Corresponding high precision data for fixed target energies are very limited especially for the antiproton projectiles. As an illustrative example Fig. 6 shows the result of the model for $\overline{\mathrm{p}} \mathrm{W}$ collisions of the E537 collaboration at $\sqrt{s}=15 \mathrm{GeV}$ [89].

The data are compared with the model calculation [90] which includes pQCD parton evolution, and the TMD effects.

The model parameters have been fixed by using data on dimuon transverse-momentum spectra in pp collisions at $\sqrt{s}=39 \mathrm{GeV}$ from the E866 collaboration in [92,93]. This model describes the dimuon production data without any adjustment of model parameters quite well, in particular, the absolute cross section in pd collisions from the E772 collaboration [94], in $\mathrm{pCu}$ collisions from the E605 collaboration [95] at the same collision energy, as well as in $\mathrm{p} A$ collisions from the E288 collaboration [96] and in pW collisions from the E439 collaboration [97] at $\sqrt{s}=27 \mathrm{GeV}$.
The presence of the abundant anti-quarks and the forward kinematics experimental arm of PANDA at the HESR-C $\bar{p} p$ collider allows to determine the minimal $M^{2}$ for which the DY mechanism works since the background contributions are expected to be surpressed in comparison to pp collisions due to the above discussed presence of the valence-anti-quarks in the $\overline{\mathrm{p}}$. (Note that the charm contribution is strongly suppressed at $x_{p} \geq 0.4$ as a lepton in a charm decay carries, on average, only $1 / 3$ of the D-meson momentum (and even less for charm baryons, and that in addition PANDA is designed to measure this contribution accurately).

The onset of factorization in the DY process with nuclei has still not been explored, but will be very interesting. Indeed, as mentioned already, the formation length in these processes is pretty small. Hence, one could think of the process in a semiclassical way, for a rather broad range of energies. In this picture, in the case of a nuclear target, the antiproton may experience one or more (in)elastic rescatterings on the target nucleons - before it annihilates with a proton into a dilepton pair. This effect can be taken into account within transport models, e.g. GiBUU [98] calculations, which are a good setup for the antiproton-nucleus dynamics. The antiproton stopping shall lead to a softening of the invariant-mass and transverse-momentum spectra of the dilepton pairs. It is also important to include these effects in future calculations, as it influences the conclusions on the in-medium modifications of the nucleon PDFs.

At high enough energies the formation time becomes large, but an antiquark which is involved in the DY process can experience energy losses growing quadratically with the path length [99]. Here one expects $p_{T}$ broadening $\propto A^{1 / 3}$. These studies, if performed as a function of the atomic number and of the collision energy at fixed $x_{\bar{q}}$, may allow to explore these important effects in great detail.

\section{Final remarks}

The exciting science discussed here could be extended to much higher energies and heavier states if - in a later phase of FAIR - one can manage to reinject antiprotons from the HESR into one or two of the higher energy main FAIR
Fig. 6 Invariant-mass (left) and transverse-momentum spectra (right) of DY dimuon pairs in $\overline{\mathrm{pW}}$ at $\sqrt{s}=15 \mathrm{GeV}$ collisions from the model described in [90] in comparison to data are from the E537 collaboration [89]. The used integrated PDF's are the MSTW2008LO68cl set [91]
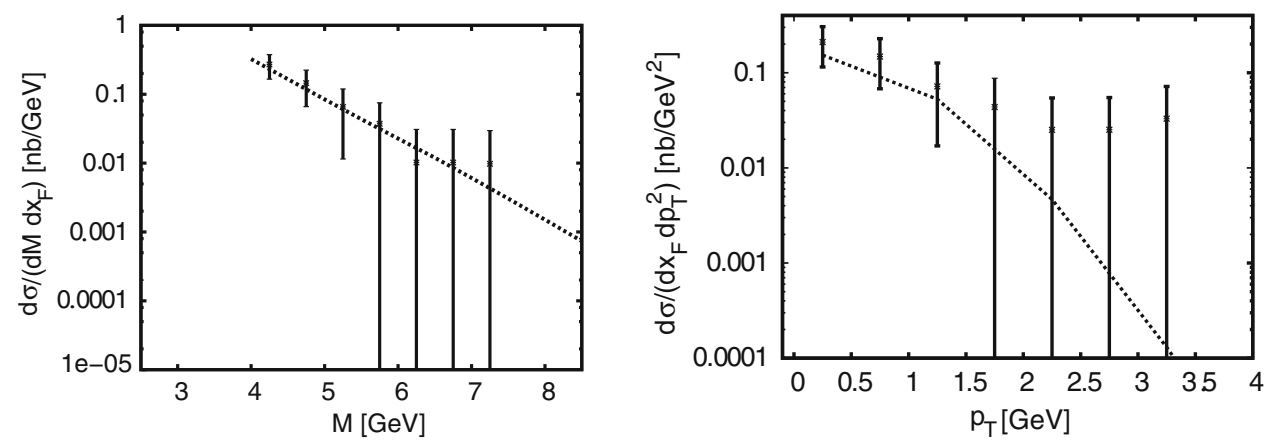
synchrotrons, SIS100 for $\sqrt{s} \leq 60 \mathrm{GeV}$ and SIS300 for $\sqrt{s} \leq 180 \mathrm{GeV} \overline{\mathrm{p}}$ collisions. Then $\overline{\mathrm{p}}$ collisions can be synchronized to run effectively and with high luminosity in a collider mode too. Also, crossing beams of SIS 100 with SIS300, e.g. $45 \mathrm{AGeV}$ heavy ions in the SIS300 colliding with $30 \mathrm{GeV}$ antiprotons (or protons), or with ions of $15 \mathrm{AGeV}$ in the SIS100 can be envisioned. Also other asymmetric collisions maybe feasible, e.g., $90 \mathrm{GeV}$ antiprotons/protons (SIS300) colliding with $15 \mathrm{AGeV}$ heavy ions (or with $30 \mathrm{GeV}$ protons or antiprotons), in the SIS100, when a common interaction region between SIS100 and SIS300 can be established.

Acknowledgements Open Access funding provided by Projekt DEAL. We thank M. Cacciari and R. Vogt for discussions on charm and beauty production in $\mathrm{p} \overline{\mathrm{p}}$ scattering, A. Motornenko for pointing at recent work on correlations as well as L. Schmitt and U. Wiedner for providing detailed information about the present status of the PANDA detector. The research by L.F. and M.S. was supported by the US Department of Energy Office of Science, Office of Nuclear Physics under Award No. DE-FG02-93ER40771. M.S. also acknowledges support by the A. von Humboldt foundation. A.L. acknowledges partial financial support by the Helmholtz International Center (HIC) for FAIR. H.v.H. acknowledges the support from Frankfurt Institute for Advanced Studies (FIAS). H.St. acknowledges the support through the Judah M. Eisenberg Laureatus Chair by Goethe University and the Walter Greiner Gesellschaft, Frankfurt. A. Larionov acknowledges support by the German Federal Ministry of Education and Research (BMBF), Grant No. 05P18RGFCA.

Data Availability Statement This manuscript has no associated data or the data will not be deposited. [Authors' comment: There are no data to be deposited related to this theoretical work.]

Open Access This article is licensed under a Creative Commons Attribution 4.0 International License, which permits use, sharing, adaptation, distribution and reproduction in any medium or format, as long as you give appropriate credit to the original author(s) and the source, provide a link to the Creative Commons licence, and indicate if changes were made. The images or other third party material in this article are included in the article's Creative Commons licence, unless indicated otherwise in a credit line to the material. If material is not included in the article's Creative Commons licence and your intended use is not permitted by statutory regulation or exceeds the permitted use, you will need to obtain permission directly from the copyright holder. To view a copy of this licence, visit http://creativecomm ons.org/licenses/by/4.0/.

\section{Appendix: PANDA detector}

The PANDA detector at FAIR can be used without modifications as a midrapidity detector in a future antiprotonproton/ion HESR-collider: primary high energy proton beams from the SIS18 at GSI are injected clockwise or counterclockwise into the HESR-C, see the dark-blue lines in Fig. 1, and collide with the $\overline{\mathrm{p}}$ 's created at the SIS100 synchrotron complex, collected in the collector ring and from there filled counterclockwise or clockwise into the High Energy Storage Ring (HESR); $\bar{p}$ and $p$ then can be simul- taneously accelerated in the magnetic fields in the HESR. The $\overline{\mathrm{p}}$ 's shall collide with the proton beam injected from SIS18 in the middle of the PANDA Detector. The PANDA collaboration focuses, in the presently planned fixed target mode with $\sqrt{s_{\max }} \sim 5.5 \mathrm{GeV}$, on hadron spectroscopy, search for exotic hadrons, hadrons in media, nucleon structure and "(multi-)hyper-, strangelets, charmed and future beauty exotic nuclei". To fully exploit PANDA and the HESR, we present the next generation physics interest for the high energy antiproton-proton collider physics, which can be realized in a highly cost effective manner by just adding one simple beam line from the existing SIS18 synchrotron to the HESR, and by using the versatile, basically unmodified PANDA detector, to provide precise trajectory reconstruction, energy and momentum measurements and an efficient particle identification system, for a momentum range from 1.5 to $15 \mathrm{GeV} / c$, with peak luminosities up to $\sim 10^{31} \mathrm{~cm}^{-2} \mathrm{~s}^{-1}$, and a number of stored antiprotons and protons, $N \sim 10^{11}$. PANDA provides nearly full coverage of the solid angle for the produced particles, together with good particle identification and high momentum $(\Delta p / p<1 \%)$ and angular resolutions for both, charged particles and photons.

PANDA is subdivided into the target spectrometer (TS), consisting of a large solenoid around the interaction region, and a forward spectrometer (FS), based on a dipole, to momentum-analyze the forward-going particles, allowing for the full angular coverage, for a wide range of energies.

The tracking systems are, from the inside outwards: the Micro-Vertex-Detector (MVD) in the TS yields a precise determination of secondary decay vertices of short-lived particles, the Straw Tube Tracker (STT) serves as central tracking device in the magnetic field providing a good momentum resolution.

A large area planar Gaseous Electron Multiplier GEMTracker provides trajectory position resolution of $<100 \mu \mathrm{m}$ for the forward going tracks in the TS.

The Forward Tracker (FT) is based on the same type of straws as the STT and is designed for momentum analysis of charged particles deflected in the field of the PANDA dipole magnet aiming at a momentum resolution better than $1 \%$.

Electromagnetic Calorimetry of PANDA allows for high count rates - the target spectrometers consist of $>15000 \mathrm{PbWO}_{4}$ crystals for an excellent pion/electron discrimination for momenta above $0.5 \mathrm{GeV} / c$ and is designed to detect photons in the energy range between $10 \mathrm{MeV}$ and $15 \mathrm{GeV}$ with a resolution $\sim 2 \%$.

The identification of charged particles with extreme accuracy is one of the key requirements for unveiling many aspects of both, the fixed target program as well as the new collider physics program envisioned here for PANDA in the decade 2030-2040. 
The PANDA Detector features dedicated particle identification (PID) systems which classify particle species over a wide kinematic range, in addition to $\mathrm{d} E / \mathrm{d} x$ measurements from tracking and information from electromagnetic calorimetry, the Detection of Internally Reflected Cherenkov light (DIRC) in the TS, a Time Of Flight System, a Muon Detection System and a Ring Imaging Cherenkov Detector in the FS.

This fully equipped PANDA Detector System, as now designed for the fixed target mode of the FAIR-MSV, is also a formidable multi-purpose instrument when used as a midrapidity HESR-collider detector:

The unchanged fixed target design's forward arm can be used for both the antiproton side - but also for the proton side in the collider mode, if the antiprotons' injection direction into HESR can be switched from upwards to downwards - and, simultaneously for the protons, from downwards to upwards.

This can be accomplished in a cost effective manner, i.e. without changing the location of the forward arm and without adding a second spectrometer, in the following manner: both injection lines into the HESR-collider, namely

- the new short proton beam line, shown in red in Fig. 1, which comes directly from the SIS 18 transfer beamline to SIS100 and

- the antiproton beam line, coming from the SIS100antiproton production target, through the $\mathrm{CR}$,

will be equipped not with only one, but with two deflector switches each, respectively, into the HESR-Collider, allowing for clockwise and counterclockwise rotation of both beams, protons and antiprotons, similar as the LHCb detector at CERN.

This enables the full HESR-C physics program-with minor adjustments to HESR-as described in the present paper-with an unmodified PANDA detector-a most costeffective proposal for realizing most exciting scientific goals.

\section{References}

1. L. Frankfurt, M. Strikman, A. Larionov, A. Lehrach, R. Maier, H. van Hees, C. Spieles, V. Vovchenko, and H. Stöcker, Novel physics opportunities at the HESR-Collider with PANDA at FAIR, to be published in FIAS Interdisciplinary Science series (2019), arXiv: 1808.09550 [hep-ph]

2. J.E. Augustin et al., (SLAC-SP-017 Collaboration), Discovery of a Narrow Resonance in $\mathrm{e}^{+} \mathrm{e}^{-}$Annihilation. Phys. Rev. Lett. 33, 1406 (1974). https://doi.org/10.1103/PhysRevLett.33.1406

3. J.J. Aubert et al., (E598 Collaboration), Experimental Observation of a Heavy Particle J. Phys. Rev. Lett. 33, 1404 (1974). https://doi. org/10.1103/PhysRevLett.33.1404
4. S. W. Herb et al., Observation of a Dimuon Resonance at $9.5 \mathrm{GeV}$ in $400 \mathrm{GeV}$ Proton-Nucleus Collisions, Phys. Rev. Lett. 39, 252 (1977), https://doi.org/10.1103/PhysRevLett.39.252

5. H. Stöcker, T. Stöhlker, C. Sturm, FAIR - cosmic matter in the laboratory. J. Phys. Conf. Ser. 623, 012026 (2015). https://doi.org/ 10.1088/1742-6596/623/1/012026

6. V. Barone et al. (PAX Collaboration), Antiproton-proton scattering experiments with polarization (2005), arXiv: hep-ex/0505054, http://arxiv.org/hep-ex/0505054

7. PAX Collaboration, Technical Proposal for AntiprotonProton Scattering Experiments with Polarization, Tech. rep., Forschungszentrum Jülich (2006), http://collaborations.fz-juelich. de/ikp/pax/public_files/proposals/techproposal20060125.pdf. Accessed Aug 2019

8. A. Lehrach, Accelerator configuration for polarized protonantiproton physics at FAIR. AIP Conf. Proc. 915, 147 (2007). https://doi.org/10.1063/1.2750755

9. I.N. Mishustin, L.M. Satarov, J. Schaffner, H. Stöcker, W. Greiner, Baryon anti-baryon pair production in strong meson fields. J. Phys. G 19, 1303 (1993). https://doi.org/10.1088/0954-3899/19/9/009

10. A.B. Larionov, I.N. Mishustin, L.M. Satarov, W. Greiner, Dynamical simulation of bound antiproton-nuclear systems and observable signals of cold nuclear compression. Phys. Rev. C 78, 014604 (2008). https://doi.org/10.1103/PhysRevC.78.014604

11. F. Bradamante, I. Koop, A. Otboev, V. Parkhomchuk, V. Reva, P. Shatunov, and Y. Shatunov, Conceptual design for a polarized proton-antiproton collider facility at GSI (2005), arXiv: physics/0511252, http://arxiv.org/abs/physics/0511252

12. A. Lehrach, O. Boine-Frankenheim, F. Hinterberger, R. Maier, D. Prasuhn, Beam performance and luminosity limitations in the highenergy storage ring (HESR). Nucl. Instrum. Meth. A 561, 289 (2006). https://doi.org/10.1016/j.nima.2006.01.017

13. P. Beller, K. Beckert, C. Dimopoulou, A. Dolinsky, F. Nolden, M. Steck, and J. Yang, Layout of an accumulator and decelerator ring for FAIR, Conf. Proc. C 060626, 199 (2006), http:// accelconf.web.cern.ch/AccelConf/e06/PAPERS/MOPCH074. PDF. Accessed Aug 2019

14. V. V. Parkhomchuk, V. B. Reva, A. N. Skrinsky, V. A. Vostrikov, K. Beckert, P. Beller, A. Dolinskii, B. Franzke, F. Nolden, and M. Steck, An Electron Cooling System for the Proposed HESR Antiproton Storage Ring, in 9th European Particle Accelerator Conference (EPAC 2004) Lucerne, Switzerland, July 5-9, 2004 (2004), http://accelconf.web.cern.ch/AccelConf/e04/ PAPERS/WEPLT056.PDF. Accessed Aug 2019

15. D. Reistad et al., Status of the HESR electron cooler design work. Conf. Proc. C 060626, 1648 (2006)

16. V. Kamerdzhiev et al., $2 \mathrm{MeV}$ Electron Cooler for COSY and HESR -First Results, in Proceedings, 5th International Particle Accelerator Conference (IPAC 2014): Dresden, Germany, June 15-20, 2014, MOPRI070 (2014), http://jacow.org/IPAC2014/papers/mopri070. pdf

17. M. Karliner, Heavy exotic quarkonia and doubly heavy baryons. EPJ Web Conf. 96, 01019 (2015). https://doi.org/10.1051/epjconf/ 20159601019

18. M. Cacciari, P. Nason, C. Oleari, A Study of heavy flavored meson fragmentation functions in $\mathrm{e}^{+} \mathrm{e}^{-}$annihilation. JHEP 04, 006 (2006). https://doi.org/10.1088/1126-6708/2006/04/006

19. M. Beneke, A. P. Chapovsky, M. Diehl, and T. Feldmann, Soft collinear effective theory and heavy to light currents beyond leading power, Nucl. Phys. B 643, 431 (2002), arXiv: hep-ph/0206152, https://doi.org/10.1016/S0550-3213(02)00687-9

20. M. Cacciari and R. Vogt, private communications

21. M. Tanabashi et al. (Particle Data Group), The Review of Particle Physics (2018), Phys. Rev. D 98, 030001 (2018), http://pdg.lbl. gov/. Accessed Aug 2019 
22. B. Blok, M. Strikman, Multiparton pp and pA collisions: from geometry to parton-parton correlations, Adv. Ser. Direct. High Energy Phys. 29, 63 (2019). arXiv: 1709.00334

23. G. Antchev et al. (TOTEM), First determination of the $\rho$ parameter at $\sqrt{s}=13 \mathrm{TeV}$ - probing the existence of a colourless three-gluon bound state (2017), arXiv: 1812.04732 [hep-ex], https://arxiv.org/ abs/1812.04732

24. G. Antchev et al. (TOTEM), Elastic differential cross-section $\mathrm{d} \sigma / \mathrm{d} t$ at $\sqrt{s}=2.76 \mathrm{TeV}$ and implications on the existence of a colourless 3-gluon bound state (2018), arXiv: 1812.08610 [hep-ex], https:// arxiv.org/abs/1812.08610

25. L. Lukaszuk, B. Nicolescu, A Possible interpretation of $\mathrm{p} p$ rising total cross-sections. Lett. Nuovo Cim. 8, 405 (1973). https://doi. org/10.1007/BF02824484

26. G.D. Alkhazov, S.L. Belostotsky, A.A. Vorobev, Scattering of 1GeV protons on nuclei. Phys. Rept. 42, 89 (1978). https://doi.org/ 10.1016/0370-1573(78)90083-2

27. A.B. Larionov, H. Lenske, Elastic scattering, polarization and absorption of relativistic antiprotons on nuclei. Nucl. Phys. A 957, 450 (2017). https://doi.org/10.1016/j.nuclphysa.2016.10.006

28. E. M. Levin and M. I. Strikman, What One May Find Out Investigating the Scattering of Hadrons from He-4?, Sov. J. Nucl. Phys. 23, 216 (1976), [Yad. Fiz.23,412(1976)]

29. I.V. Moskalenko, A.W. Strong, J.F. Ormes, M.S. Potgieter, Secondary anti-protons and propagation of cosmic rays in the galaxy and heliosphere. Astrophys. J. 565, 280 (2002). https://doi.org/10. $1086 / 324402$

30. A.A. Tyapkin, A possible way of establishing the existence of charmed particles. Sov. J. Nucl. Phys. 22, 89 (1976)

31. C.B. Dover, S.H. Kahana, Possibility of charmed hypernuclei. Phys. Rev. Lett. 39, 1506 (1977). https://doi.org/10.1103/ PhysRevLett.39.1506

32. K. Tsushima, F.C. Khanna, Lambda(c)+ and Lambda(b) hypernuclei. Phys. Rev. C 67, 015211 (2003). https://doi.org/10.1103/ PhysRevC.67.015211

33. A.B. Larionov, H. Lenske, Distillation of scalar exchange by coherent hypernucleus production in antiproton-nucleus collisions. Phys. Lett. B 773, 470 (2017). https://doi.org/10.1016/j. physletb.2017.09.007

34. R. Shyam, K. Tsushima, Production of $\Lambda_{c}^{+}$hypernuclei in antiproton-nucleus collisions. Phys. Lett. B 770, 236 (2017). https://doi.org/10.1016/j.physletb.2017.04.057

35. L. Gerland, L. Frankfurt, M. Strikman, H. Stöcker, and W. Greiner, $\mathrm{J} / \psi$ production, $\chi$ polarization and color fluctuations, Phys. Rev. Lett. 81, 762 (1998), arXiv:nucl-th/9803034, https://doi.org/10. 1103/PhysRevLett.81.762

36. D. Dutta, K. Hafidi, M. Strikman, Color transparency: past, present and future. Prog. Part. Nucl. Phys. 69, 1 (2013). https://doi.org/10. 1016/j.ppnp.2012.11.001

37. L.L. Frankfurt, M.I. Strikman, Point-like configurations in hadrons and nuclei and deep inelastic reactions with leptons: EMC and EMC-like effects. Nucl. Phys. B 250, 143 (1985). https://doi.org/ 10.1016/0550-3213(85)90477-8

38. M. Alvioli, B.A. Cole, L. Frankfurt, D.V. Perepelitsa, M. Strikman, Evidence for $x$-dependent proton color fluctuations in $\mathrm{pA}$ collisions at the CERN large hadron collider. Phys. Rev. C 93, 011902 (2016). https://doi.org/10.1103/PhysRevC.93.011902

39. M. Alvioli, L. Frankfurt, D. Perepelitsa, M. Strikman, Global analysis of color fluctuation effects in proton- and deuteron-nucleus collisions at RHIC and the LHC. Phys. Rev. D 98, 071502 (2018). https://doi.org/10.1103/PhysRevD.98.071502

40. H.A. Bethe, Nuclear many-body problem. Phys. Rev. 103, 1353 (1956). https://doi.org/10.1103/PhysRev.103.1353

41. J. Goldstone, Derivation of the Brueckner Many-Body Theory. Proc. Roy. Soc. Lond. A 239, 267 (1957). https://doi.org/10.1098/ rspa.1957.0037
42. K.A. Brueckner, J.L. Gammel, Properties of nuclear matter. Phys. Rev. 109, 1023 (1958). https://doi.org/10.1103/PhysRev.109.1023

43. M.I. Gorenstein, G.M. Zinovev, Cluster model of cumulative particle production in hadron-nucleus collisions. Phys. Lett. B 67, 100 (1977). https://doi.org/10.1016/0370-2693(77)90817-6

44. A. Motornenko, M.I. Gorenstein, Cumulative production of pions by heavy baryonic resonances in proton-nucleus collisions. J. Phys. G 44, 025105 (2017). https://doi.org/10.1088/1361-6471/aa51f6

45. O. Panova, A. Motornenko, M.I. Gorenstein, J. Steinheimer, H. Stoecker, Backward nucleon production by heavy baryonic resonances in proton-nucleus collisions. Phys. Rev. C 100, 054617 (2019). https://doi.org/10.1103/PhysRevC.100.054617

46. L.L. Frankfurt, M.I. Strikman, How to treat short range nucleon correlations in high-energy lepton or hadron scattering off deuteron nuclei. Phys. Lett. 69B, 93 (1977). https://doi.org/10. 1016/0370-2693(77)90141-1

47. L.L. Frankfurt, M.I. Strikman, High-energy phenomena, short range nuclear structure and QCD. Phys. Rept. 76, 215 (1981). https://doi.org/10.1016/0370-1573(81)90129-0

48. L.L. Frankfurt, M.I. Strikman, D.B. Day, M. Sargsian, Evidence for short range correlations from high $\mathrm{Q}^{* * 2}$ (e, e-prime) reactions. Phys. Rev. C 48, 2451 (1993). https://doi.org/10.1103/PhysRevC. 48.2451

49. E. Piasetzky, M. Sargsian, L. Frankfurt, M. Strikman, J.W. Watson, Evidence for the strong dominance of proton-neutron correlations in nuclei. Phys. Rev. Lett. 97, 162504 (2006). https://doi.org/10. 1103/PhysRevLett.97.162504

50. M. Duer et al., (CLAS), direct observation of proton-neutron shortrange correlation dominance in heavy nuclei. Phys. Rev. Lett. 122, 172502 (2019). https://doi.org/10.1103/PhysRevLett.122.172502

51. R.G. Arnold, C.E. Carlson, F. Gross, Elastic electron-deuteron scattering at high-energy. Phys. Rev. C 21, 1426 (1980). https:// doi.org/10.1103/PhysRevC.21.1426

52. T. De Forest, Off-shell electron nucleon cross-sections. the impulse approximation, Nucl. Phys. A 392, 232 (1983), https://doi.org/10. 1016/0375-9474(83)90124-0

53. M. M. Sargsian, T. V. Abrahamyan, M. I. Strikman, and L. L. Frankfurt, Exclusive electro-disintegration of ${ }^{3} \mathrm{He}$ at high $Q^{2}$. II. Decay function formalism, Phys. Rev. C 71, 044615 (2005), https:// doi.org/10.1103/PhysRevC.71.044615

54. D. B. Day, L. L. Frankfurt, M. M. Sargsian, and M. I. Strikman, Towards observation of three-nucleon short-range correlations in high $Q^{2} A\left(\mathrm{e}, \mathrm{e}^{\prime}\right) X$ reactions (2018), arXiv: 1803.07629 [nucl-th], https://arxiv.org/abs/1803.07629

55. L. Van Hove, S. Pokorski, High-energy hadron-hadron collisions and internal hadron structure. Nucl. Phys. B 86, 243 (1975). https:// doi.org/10.1016/0550-3213(75)90443-5

56. S. Raha, Dilepton, diphoton and photon production in preequilibrium. Phys. Scripta T32, 180 (1990). https://doi.org/10.1088/ 0031-8949/1990/T32/030

57. E.V. Shuryak, Two stage equilibration in high-energy heavy ion collisions. Phys. Rev. Lett. 68, 3270 (1992). https://doi.org/10.1103/ PhysRevLett.68.3270

58. J. Alam, B. Sinha, S. Raha, Successive equilibration in quark-gluon plasma. Phys. Rev. Lett. 73, 1895 (1994). https://doi.org/10.1103/ PhysRevLett.73.1895

59. T.S. Biro, E. van Doorn, B. Muller, M.H. Thoma, X.N. Wang, Parton equilibration in relativistic heavy ion collisions. Phys. Rev. C 48, 1275 (1993). https://doi.org/10.1103/PhysRevC.48.1275

60. D.M. Elliott, D.H. Rischke, Chemical equilibration of quarks and gluons at RHIC and LHC energies. Nucl. Phys. A 671, 583 (2000). https://doi.org/10.1016/S0375-9474(99)00840-4

61. Z. Xu, C. Greiner, Thermalization of gluons in ultrarelativistic heavy ion collisions by including three-body interactions in a parton cascade. Phys. Rev. C 71, 064901 (2005). https://doi.org/10. 1103/PhysRevC.71.064901 
62. A. Kurkela, A. Mazeliauskas, Chemical equilibration in weakly coupled QCD. Phys. Rev. D 99, 054018 (2019). https://doi.org/10. 1103/PhysRevD.99.054018

63. A. Kurkela, A. Mazeliauskas, Chemical equilibration in hadronic collisions, Phys. Rev. Lett. 122, 142301 (2019). https://doi.org/10. 1103/PhysRevLett.122.142301

64. P.B. Arnold, G.D. Moore, L.G. Yaffe, Effective kinetic theory for high temperature gauge theories. JHEP 01, 030 (2003). hep$\mathrm{ph} / 0209353$

65. A. Kurkela, Y. Zhu, Isotropization and hydrodynamization in weakly coupled heavy-ion collisions. Phys. Rev. Lett. 115, 182301 (2015). https://doi.org/10.1103/PhysRevLett.115.182301

66. M.P. Heller, A. Kurkela, M. Spaliski, V. Svensson, Hydrodynamization in kinetic theory: transient modes and the gradient expansion. Phys. Rev. D 97, 091503 (2018). https://doi.org/10. 1103/PhysRevD.97.091503

67. H. Stöcker et al., Glueballs amass at RHIC and LHC Colliders! the early quarkless 1 st order phase transition at $T=270 \mathrm{MeV}$ from pure Yang-Mills glue plasma to GlueBall-Hagedorn states. J. Phys. G 43, 015105 (2016). https://doi.org/10.1088/0954-3899/ $43 / 1 / 015105$

68. H. Stöcker et al., Under'saturation of quarks at early stages of relativistic nuclear collisions: The hot glue initial scenario and its observable signatures. Astron. Nachr. 336, (2015). https://doi.org/ 10.1002/asna.201512252

69. S. Borsanyi, G. Endrodi, Z. Fodor, S.D. Katz, K.K. Szabo, Precision SU(3) lattice thermodynamics for a large temperature range. JHEP 07, 056 (2012). https://doi.org/10.1007/JHEP07(2012)056

70. M. Beitel, C. Greiner, H. Stöcker, Fast dynamical evolution of a hadron resonance gas via Hagedorn states. Phys. Rev. C 94, 021902 (2016). https://doi.org/10.1103/PhysRevC.94.021902

71. V. Vovchenko, M.I. Gorenstein, L.M. Satarov, I.N. Mishustin, L.P. Csernai, I. Kisel, H. Stöcker, Entropy production in chemically nonequilibrium quark-gluon plasma created in central $\mathrm{Pb}+\mathrm{Pb}$ collisions at energies available at the CERN Large Hadron Collider. Phys. Rev. C 93, 014906 (2016). https://doi.org/10.1103/ PhysRevC.93.014906

72. V. Vovchenko, I.A. Karpenko, M.I. Gorenstein, L.M. Satarov, I.N. Mishustin, B. Kämpfer, H. Stöcker, Electromagnetic probes of a pure-glue initial state in nucleus-nucleus collisions at energies available at the CERN Large Hadron Collider. Phys. Rev. C 94, 024906 (2016). https://doi.org/10.1103/PhysRevC.94.024906

73. V. Vovchenko, L.-G. Pang, H. Niemi, I. A. Karpenko, M. I. Gorenstein, L. M. Satarov, I. N. Mishustin, B. Kämpfer, and H. Stöcker, Hydrodynamic modeling of a pure-glue initial scenario in highenergy hadron and heavy-ion collisions, PoS BORMIO2016, 039 (2016), https://doi.org/10.22323/1.272.0039

74. I. Karpenko, P. Huovinen, M. Bleicher, A 3+1 dimensional viscous hydrodynamic code for relativistic heavy ion collisions. Comput. Phys. Commun. 185, 3016 (2014). https://doi.org/10.1016/j.cpc. 2014.07.010

75. S. Borsanyi, Z. Fodor, C. Hoelbling, S.D. Katz, S. Krieg, K.K. Szabo, Full result for the QCD equation of state with $2+1$ flavors. Phys. Lett. B 730, 99 (2014). https://doi.org/10.1016/j.physletb. 2014.01.007

76. V. Vovchenko, Quantum statistical van der Waals equation and its QCD applications, Ph.D. thesis, Goethe University Frankfurt (2018), http://publikationen.ub.uni-frankfurt.de/frontdoor/index/ index/docId/46301. Accessed Aug 2019

77. R. Rapp, J. Wambach, Low mass dileptons at the CERN SPS: Evidence for chiral restoration? Eur. Phys. J. A 6, 415 (1999). https:// doi.org/10.1007/s100500050364

78. R. Rapp, J. Wambach, H. van Hees, The chiral restoration transition of QCD and low mass dileptons. Landolt-Börnstein 23, 134 (2010). https://doi.org/10.1007/978-3-642-01539-7_6
79. S. Endres, H. van Hees, M. Bleicher, Photon and dilepton production at the facility for proton and anti-proton research and beam-energy scan at the relativistic heavy-ion collider using coarse-grained microscopic transport simulations. Phys. Rev. C 93, 054901 (2016). https://doi.org/10.1103/PhysRevC.93.054901

80. T. Galatyuk, P.M. Hohler, R. Rapp, F. Seck, J. Stroth, Thermal dileptons from coarse-grained transport as fireball probes at SIS energies. Eur. Phys. J. A 52, 131 (2016). https://doi.org/10.1140/ epja/i2016-16131-1

81. J. Staudenmaier, J. Weil, V. Steinberg, S. Endres, H. Petersen, Dilepton production and resonance properties within a new hadronic transport approach in the context of the GSI-HADES experimental data. Phys. Rev. C 98, 054908 (2018). https://doi. org/10.1103/PhysRevC.98.054908

82. O. Linnyk, E.L. Bratkovskaya, W. Cassing, Effective QCD and transport description of dilepton and photon production in heavyion collisions and elementary processes. Prog. Part. Nucl. Phys. 87, 50 (2016). https://doi.org/10.1016/j.ppnp.2015.12.003

83. S. Dulat, T.-J. Hou, J. Gao, M. Guzzi, J. Huston, P. Nadolsky, J. Pumplin, C. Schmidt, D. Stump, C.-P. Yuan, New parton distribution functions from a global analysis of quantum chromodynamics. Phys. Rev. D 93, 033006 (2016). https://doi.org/10.1103/ PhysRevD.93.033006

84. M. Glück, E. Reya, and I. Schienbein, Pionic parton distributions revisited, Eur. Phys. C 10, 313 (1999), http://doi.org/10.1007/ s100529900124

85. C. Spieles, L. Gerland, N. Hammon, M. Bleicher, S.A. Bass, H. Stöcker, W. Greiner, C. Lourenco, R. Vogt, A Microscopic calculation of secondary Drell-Yan production in heavy ion collisions. Eur. Phys. J. C 5, 349 (1998). https://doi.org/10.1007/ s100520050279

86. C. Spieles, L. Gerland, N. Hammon, M. Bleicher, S.A. Bass, H. Stöcker, W. Greiner, C. Lourenco, R. Vogt, Intermediate mass dileptons from secondary Drell-Yan processes. Nucl. Phys. A 638 , 507 (1998). https://doi.org/10.1016/S0375-9474(98)00345-5

87. C. Spieles, M. Bleicher, Secondary Drell-Yan dileptons in heavyion reactions. Int. J. Mod. Phys. E 28, 1950047 (2019). https://doi. org/10.1142/S0218301319500472

88. R. Angeles-Martinez et al., Transverse momentum dependent (TMD) parton distribution functions: status and prospects. Acta Phys. Polon. B 46, 2501 (2015). https://doi.org/10.5506/ APhysPolB.46.2501

89. E. Anassontzis et al., High mass dimuon production in $\bar{p} n$ and $\pi^{-} n$ interactions at 125-GeV/c. Phys. Rev. D 38, 1377 (1988). https:// doi.org/10.1103/PhysRevD.38.1377

90. F. Eichstaedt, S. Leupold, K. Gallmeister, H. van Hees, and U. Mosel, Description of Fully Differential Drell-Yan Pair Production, PoS BORMIO2011, 042 (2011), arXiv: 1108.5287 [hep-ph], https://arxiv.org/abs/1108.5287

91. A.D. Martin, W.J. Stirling, R.S. Thorne, G. Watt, Parton distributions for the LHC. Eur. Phys. J. C 63, 189 (2009). https://doi.org/ 10.1140/epjc/s10052-009-1072-5

92. J. C. Webb et al. (NuSea Collaboration), Absolute Drell-Yan dimuon cross-sections in $800 \mathrm{GeV} / c$ pp and pd collisions (2003), arXiv: hep-ex/0302019, https://arxiv.org/abs/hep-ex/0302019

93. J. C. Webb, Measurement of continuum dimuon production in $800 \mathrm{GeV} / \mathrm{c}$ proton nucleon collisions, Ph.D. thesis, New Mexico State U. (2003), arXiv: hep-ex/0301031, https://doi.org/10.2172/ 1155678

94. P. L. McGaughey et al. (E772 Collaboration), Cross-sections for the production of high mass muon pairs from 800$\mathrm{GeV}$ proton bombardment of H-2, Phys. Rev. D 50, 3038 (1994), [Erratum: Phys. Rev.D60,119903(1999)], https://doi.org/ 10.1103/PhysRevD.50.3038, https://doi.org/10.1103/PhysRevD. 60.119903 
95. G. Moreno et al., Dimuon production in proton - copper collisions at $\sqrt{s}=38.8 \mathrm{GeV}$, Phys. Rev. D 43, 2815 (1991), https://doi.org/ 10.1103/PhysRevD.43.2815

96. A.S. Ito et al., Measurement of the continuum of dimuons produced in high-energy proton-nucleus collisions. Phys. Rev. D 23, 604 (1981). https://doi.org/10.1103/PhysRevD.23.604

97. S.R. Smith et al., Experimental test of the Drell-Yan model in $p W \rightarrow \mu^{+} \mu^{-} X$. Phys. Rev. Lett. 46, 1607 (1981). https://doi. org/10.1103/PhysRevLett.46.1607
98. O. Buss, T. Gaitanos, K. Gallmeister, H. van Hees, M. Kaskulov et al., Transport-theoretical description of nuclear reactions. Phys. Rept. 512, 1 (2012). https://doi.org/10.1016/j.physrep.2011.12. 001

99. R. Baier, Y.L. Dokshitzer, A.H. Mueller, S. Peigne, D. Schiff, Radiative energy loss and $\mathrm{p}(\mathrm{T})$ broadening of high-energy partons in nuclei. Nucl. Phys. B 484, 265 (1997). https://doi.org/10.1016/ S0550-3213(96)00581-0 